Oscar W. Gabriel

\title{
Lokale Politische Kultur
}

\section{Inhalt}

1. Das Untersuchungsproblem: Lokale Demokratie und Politische Kultur . . . . . . . . . . . . . . . . . . . . 203

2. Der Begriff "Politische Kultur" . . . . . . . . . . . . . . . . . 204

2.1. Politische Kultur als wissenschaftlicher Untersuchungsgegenstand . . 204

2.2. Der wissenschaftstheoretische Hintergrund der Erforschung

2.3. Der Gegenstand der Erforschung Politischer Kultur -

Der enge und der weite Begriff Politischer Kultur . . . . . . . . . . . 205

2.4. Ein Raster zur Beschreibung Politischer Kulturen . . . . . . . . . . . 206

2.4.1. Orientierungsarten und Orientierungsobjekte . . . . . . . . . 206

2.4.2. Die Verteilung der Orientierungen:

Homogene und gespaltene Politische Kulturen . . . . . . . . . . .208

2.4.3. Die Staatsbürgerkultur als Politische Kultur der Demokratie . . . . . . 209

3. Lokale Politische Kultur . . . . . . . . . . . . . . . . . . . 210

3.1. Forschungsrichtungen, Datenlage und methodisches Vorgehen . . . . 210

3.2. Verwendungsweisen des Begriffs lokale Politische Kultur . . . . . . . 212

3.2.1. Lokale Politische Kultur als System von Einstellungen zur lokalen Politik . . . . . . . . . . . . . . . . . . . . . . 212

3.2.2. Lokale Zugehörigkeit als Erklärungsfaktor Politischer Kultur . . . . . 213

4. Geschichtliche Rahmenbedingungen der lokalen Politischen Kultur in der Bundesrepublik Deutschland . . . . . . . . . . . . . 214

5. Die lokale Politische Kultur der Bundesrepublik Deutschland . . . 216

5.1. Die allgemeine Beziehung der Bevölkerung zur Gemeinde und zur Kommunalpolitik . . . . . . . . . . . . . . . . . . . . 216

5.1.1. Die Gemeindeidentifikation . . . . . . . . . . . . . . . . . . 216

5.1.2. Das Interesse an der Kommunalpolitik, das subjektive Verständnis kommunaler Fragen und das kommunalpolitische Wissen . . . . . . 219

5.2. Kommunalpolitik zwischen Parteipolitik und Verwaltung . . . . . . 223

5.2.1. Die Einschätzung der "Politikfähigkeit" der kommunalen Selbstverwaltung und der Rolle der Parteien in der Kommunalpolitik . . . . 223

5.2.2. Die Einstellungen zur Kommunalverwaltung . . . . . . . . . . . 225

5.2.3. Politische Institutionen, Parteipolitik und Verwaltung aus der Sicht des lokalpolitischen Führungspersonals . . . . . . . . . . 233

5.3. Partizipative Orientierungen: Politische Kompetenz und

5.4. Differenzierungsmerkmale: Gibt es lokale politische Subkulturen? . . 241 
6. Zusammenfassung und SchluBfolgerungen . . . . . . . . . . . . 244

6.1. Charakteristika der lokalen Politischen Kultur der Bundesrepublik . . 244

6.2. Lokale Politische Kultur und kommunale Demokratie -

Zur systematischen Bedeutung politisch-kultureller Faktoren für die

Funktionsweise des politischen Systems . . . . . . . . . . . . .245

Literatur . . . . . . . . . . . . . . . . . . . . 247 


\section{Das Untersuchungsproblem: Lokale Demokratie und Politische Kultur ${ }^{1}$}

Als Beobachter des kommunalpolitischen Geschehens stellt man immer wieder fest, daß zwischen Gemeinden mit der gleichen Kommunalverfassung und einer ähnlichen Sozialstruktur beträchtliche politische Unterschiede bestehen. So räumt das Kommunalwahlrecht den Wählerinnen und Wählern in Bayern und Baden-Württemberg einen wesentlich größeren Einfluß auf die personelle Zusammensetzung der Kommunalvertretung ein als in Nordrhein-Westfalen. Dennoch liegt die Beteiligung an den Stadt- und Gemeinderatswahlen in Bayern traditionell höher als in Baden-Württemberg, während in Nordrhein-Westfalen keineswegs eine unterdurchschnittliche Wahlbeteiligung zu verzeichnen ist. Die Schwankungen in den Stimmenanteilen der politischen Parteien bei Kommunalwahlen lassen sich ebenfalls nicht ausschlieBlich auf Unterschiede in der Sozialstruktur der betreffenden Gemeinden zurückführen. Wie die spektakulären Wahlerfolge der SPD bei den Oberbürgermeisterwahlen 1990 in Passau und Regensburg zeigen, führt ein hoher Katholikenanteil nicht zwangsläufig zu einer Vorherrschaft der CDU/CSU. Ebenso wenig garantiert ein hoher Arbeiteranteil klare SPD-Mehrheiten in den kommunalen Vertretungskörperschaften.

Auch andere Merkmale der kommunalpolitischen Struktur wandeln sich im Zeitablauf, ohne daß man dies in jedem Falle auf Veränderungen der Sozialstruktur oder des politischen Institutionensystems zurückführen könnte. Die seit der Mitte der sechziger Jahre stark zunehmende politische Aktivität der Bevölkerung wurde kaum durch die gemeinderechtlich normierten Beteiligungsmöglichkeiten beeinfluBt: In baden-württembergischen Kommunen, in denen die Bevölkerung bereits zum damaligen Zeitpunkt über umfassende Partizipationsrechte verfügte, nahm die politische Partizipation ebenso zu wie in Schleswig-Holstein, in dessen Kommunen die Teilnahmemöglichkeiten bis vor kurzem auf die Stimmabgabe bei Kommunalwahlen beschränkt waren.

Die Suche nach den Ursachen der beschriebenen Unterschiede führt zum Faktor "lokale Politische Kultur"2, d.h. zu den Wertorientierungen und Einstellungen der Bevölkerung. Diese werden durch Ereignisse im lokalen Umfeld oder durch örtliche Traditionen geprägt und können die Funktionsweise des politischen Systems einer Gemeinde erheblich beeinflussen. Die Eigenschaften der lokalen Politischen Kultur der Bundesrepublik Deutschland und ihre Bedeutung für die Analyse kommunalpolitischer Strukturen und Prozesse bilden den Gegenstand dieser Untersuchung.

1 Die in diesem Beitrag benutzten Daten wurden vom Zentralarchiv für empirische Sozialforschung, Universität zu Köln, zugänglich gemacht. Sie wurden von verschiedenen Primärforschern erhoben und vom Zentralarchiv für empirische Sozialforschung für die Analyse aufbereitet und dokumentiert. Weder die Primärforscher noch das Zentralarchiv tragen irgendeine Verantwortung für die Analyse und Interpretation der Daten in diesem Beitrag.

2 Die Begriffe "kommunal" und "lokal" werden in den folgenden Ausfuhrungen austauschbar benutzt. 


\section{Der Begriff "Politische Kultur"}

\subsection{Politische Kultur als wissenschaftlicher Untersuchungsgegenstand}

Die wissenschaftliche Auseinandersetzung mit dem Gegenstand "Politische Kultur" ist nicht neu: Bereits in der Antike untersuchte man die kulturellen Voraussetzungen bestimmter Herrschaftsformen. Mit der Nationalcharakterforschung und der Völkerpsychologie entwickelten sich im 19. Jahrhundert Vorläuferdisziplinen der modernen Erforschung Politischer Kultur. Weitere Impulse emielt dieser Forschungszweig durch die soziologische Theorie des 20. Jahrhunderts. Erwähnung verdienen in diesem Zusammenhang vor allem die Arbeiten von Max Weber und Talcott Parsons, die empirische Meinungsforschung, die Persönlichkeits- und Sozialpsychologie sowie die Wahlsoziologie der Ann Arbor- und der Columbia-Schule (ausführlicher: ALMOND 1980; BERG-SCHLOSSER/SCHISSLER 1987).

Seit seiner Einführung in die Politikwissenschaft (zuerst: ALMOND 1956) durchlief der Begriff der Politischen Kultur eine bemerkenswerte Karriere. Mittlerweile ist er aus der Politikwissenschaft, der politischen Publizistik und aus der praktischen Politik kaum noch wegzudenken. In der Alltagssprache verwendet man den Begriff vorzugsweise in bewertender Absicht, vor allem um das eigene Verhalten positiv, das des politischen Gegners aber negativ darzustellen. Die Begriffe "Politische Kultur" und "politischer Stil" sind dabei weitgehend bedeutungsgleich, und beide werden nicht näher bestimmt. Während diese inhaltliche Unbestimmtheit in der Alltagssprache noch angehen mag, hat die Wissenschaftssprache den Begriff und die durch ihn bezeichneten Sachverhalte präzise zu bestimmen. In dieser Hinsicht läßt die gegenwärtige Forschung viele Wünsche offen. Gerade weil sich die Unterschiede in der Verwendung des Begriffes "Politische Kultur" nicht völlig ausräumen lassen, ist es notwendig, seine verschiedenartigen Verwendungsweisen $\mathrm{zu}$ charakterisieren und ihre theoretischen und forschungspraktischen Folgen zu prüfen.

\subsection{Der wissenschaftstheoretische Hintergrund der Erforschung Politischer Kultur - normative und empirische Ansätze}

Bei der Verwendung des Begriffes "Politische Kultur" steht jeder Forscher vor der Entscheidung zwischen einer beschreibenden und einer bewertenden Vorgehensweise. Dieser Gegensatz zwischen einer empirisch und einer normativ ausgerichteten Erforschung Politischer Kultur ist nicht ausräumbar, weil er auf grundsätzlichen Auffassungsunterschieden über die methodologische Orientierung unseres Faches beruht. Normativ orientierte Forscher binden ihre Konzeption Politischer Kultur in eine bestimmten politischen Werten verpflichtete Demokratietheorie ein. So verweisen beispielsweise REICHEL (1981) und JARREN et al. (1989) auf die partizipative Demokratietheorie als Wertbasis ihrer Untersuchungen. Dementsprechend erheben sie das Ausmaß und die Qualität politischer Beteiligung zum Maßstab für die Beurteilung der politischen Wirklichkeit. Im Gegensatz dazu bettet Graf KIELMANSEGG (1989) den Begriff der Politischen Kultur in ein repräsentativ-demokratisches Verfassungsverständnis ein und gewinnt auf diese Weise andere Maßstäbe für eine kritische Auseinandersetzung mit der Demokratie in der Bundesrepublik. Die normative Bedeutung der Begriffe "Politische Kultur" und "demokratische Politische Kultur" steht somit nicht fest, sondern sie ergibt sich aus dem demokratietheoretischen Zusammenhang, 
in den sie eingebunden sind. Dies kann dazu führen, daß relativ gleichartige $\mathrm{Be}-$ schreibungen des politischen Status Quo mit sehr unterschiedlichen Bewertungen und praktischen Empfehlungen einhergehen.

Im Gegensatz dazu weisen empirisch orientierte Wissenschaftler der Erforschung Politischer Kultur ausschließlich die Aufgabe zu, die politische Wirklichkeit zu beschreiben und zu erklären. Sie betrachten die Festlegung von WertmaBstäben als Aufgabe der praktischen Politik oder der Verfassung, nicht der Wissenschaft. Nachfolgend findet das durch die empirische Wissenschaftslehre geprägte Konzept Politischer Kultur Verwendung, d.h. es geht um die Beschreibung und Erklärung eines bestimmten Ausschnitts aus der politischen Wirklichkeit, nicht um deren Bewertung. Dies schlieBt natürlich nicht aus, daß bei der Auswahl von Untersuchungsproblemen normative Überlegungen eine Rolle spielen.

\subsection{Der Gegenstand der Erforschung Politischer Kultur - Der enge und der weite Begriff Politischer Kultur}

Das zweite Konfliktfeld betrifft den Gegenstandsbereich der Erforschung Politischer Kultur. In dieser Hinsicht kann man zwischen einem engen und einem weiten Begriff Politischer Kultur unterscheiden. Das enge Konzept steht in der Tradition von ALMOND und VERBA, die als Begründer der Erforschung Politischer Kultur anzusehen sind. ALMOND/VERBA (1965: 13) bezeichneten die Verteilung individueller Einstellungen zu politischen Objekten unter den Mitgliedern eines Kollektivs oder die subjektive, die psychologische Dimension der Politik als Politische Kultur (ausführlicher zu diesem Ansatz siehe PATRICK 1984: 273 ff.). Dem Begriff der Einstellungen auf der Individualebene entspricht der Begriff der Kultur auf der Kollektivebene.

Aus der Kritik an der angeblichen Begrenztheit dieses Ansatzes ergab sich die Forderung nach einem weiteren Begriff, wie sie kürzlich JARREN et al. (1989: 44 ff.) erhoben. Vertreter des weiten oder umfassenden Ansatzes sprechen sich dafür aus, neben den politischen Einstellungen die politischen Institutionen, historische Entwicklungsprozesse, Sozialisationsprozesse und vor allem das politische Verhalten zum Gegenstand der Erforschung Politischer Kultur zu machen (vgl. weitere Hinweise bei PATRICK 1984: 279 ff.). So beschreibt z.B. SONTHEIMER (1985: 99 f.), den Gegenstandsbereich "Politische Kultur" wie folgt:

"Man kann die Politische Kultur eines Systems nicht allein von der subjektiven Seite aus erfassen, sondern muß auch die objektiven Gegebenheiten und historischen Prozesse berücksichtigen, die für die Entstehung der subjektiven Einstellungen und Gefühlshaltungen oft von entscheidender Bedeutung sind. Die Politische Kultur der Bundesrepublik ist also einerseits erkennbar in den Einstellungen der Bürger gegenuber der politischen Ordnung, ihren Trägern und Leistungen, andererseits ist diese Ordnung selbst ein maßgeblicher Teil der Politischen Kultur, denn in ihr verkörpern sich Werthaltungen, in ihr prägen sich mehr oder weniger typische Verhaltensweisen aus, denen man mindestens so viel Gewicht beimessen muß wie den subjektiven Reaktionen der Gesellschaftsmitglieder auf die Institutionen, ihr Verhalten und dessen Auswirkungen" (Hervorh. OWG.).

Gegen diese weite Verwendung des Begriffs "Politische Kultur" sprechen mindestens zwei Gründe. Abgesehen davon, daß die Begriffe Politik und Politische Kultur in dieser Konzeption deckungsgleich werden und somit einer von ihnen unter dem Gesichtspunkt der Forschungsökonomie überflüssig ist, beruht die Forderung, den $\mathrm{Be}$ griff "Politische Kultur" auf alle möglichen Wirklichkeitsausschnitte auszuweiten, auf 
einer Vermischung von Beschreibungs- und Erklärungsproblemen. Mit der Eingrenzung des Kulturbegriffs auf die Verteilung von Einstellungen werden die politischen Institutionen, das politische Verhalten oder die politischen Entwicklungsprozesse nicht zwangsläufig aus dem Objektbereich der Analyse Politischer Kultur ausgeklammert. Je nachdem, mit welchem konkreten Forschungsproblem man sich beschäftigt, behandelt man diese Größen vielmehr als Produkte oder als Bestimmungsfaktoren Politischer Kultur. Bereits in "The Civic Culture" stellten ALMOND und VERBA (1965) die Politische Kultur eines Landes als Ergebnis historischer Entwicklungen dar und sahen in ihr einen bedeutsamen Bestimmungsfaktor der Stabilität und Leistungsfähigkeit der politischen Institutionen. Die Rolle politisch-kultureller Größen in einer Theorie politischer Systeme läßt sich nur dann einwandfrei klären, wenn man die zu erklärenden und die erklärenden Variablen begrifflich klar voneinander abgrenzt (vgl. hierzu die vorzügliche Untersuchung von LIJPHART 1980).

Aus den genannten Gründen liegt der folgenden Untersuchung das engere, einstellungsorientierte Konzept Politischer Kultur zugrunde. Der Terminus Politische Kultur dient als Bezeichnung für die Verteilung politischer Einstellungen und wird von den politischen Strukturen und vom politischen Verhalten unterschieden. Er enthält die folgenden Definitionselemente:

(1) Die Politische Kultur eines Kollektivs umfaßt die Einstellungen seiner Mitglieder.

(2) Diese Einstellungen beziehen sich auf politische Objekte. Durch ihren Objektbezug unterscheidet sich die Politische Kultur von der allgemeinen Kultur einer Gesellschaft und von der Kultur anderer gesellschaftlicher Teilbereiche, z.B. der Wirtschaft, der Religion oder der Freizeitgestaltung.

(3) Politische Kollektive wie Staaten, Gemeinden, Verbände oder soziale Schichten weisen spezifische Einstellungsmuster - also eine Politische Kultur - auf, durch die sie sich von anderen Kollektiven unterscheiden.

(4) Die Einstellungen der Kollektivmitglieder sind nicht homogen, vielmehr weist jedes Kollektiv eine bestimmte Verteilung politischer Einstellungen auf.

Die Beschaffenheit der Politischen Kultur eines Landes oder einer Gemeinde und ihre Beziehung zu anderen Aspekten des gesellschaftlichen und politischen Lebens ist von der empirischen Forschung zu ermitteln.

\subsection{Ein Raster zur Beschreibung Politischer Kulturen}

\subsubsection{Orientierungsarten und Orientierungsobjekte}

Die vorgeschlagene Definition grenzt die Politische Kultur nicht nur von politischen Institutionen, Organisationen und Verhaltensmustern ab. Sie eignet sich darüber hinaus als Grundlage für die Beschreibung konkreter Politischer Kulturen. ALMOND und VERBA (1965: 14 ff.) entwickelten auf der Grundlage einer Kombination von Orientierungsarten und Orientierungsobjekten ein Beschreibungsraster und eine Typologie Politischer Kultur, die sich in vereinfachter Form auch für unsere Zwecke eignet (ausführlich hierzu: DIAS 1971; GABRIEL 1986: 38 ff.). Als Orientierungsarten führten sie politische Kenntnisse, Gefühle und Wertorientierungen ein. Da diese Abgrenzung forschungspraktisch kaum umsetzbar ist und zudem für ALMOND/VERBAS Modell Politischer Kultur keine Rolle spielt, wird sie hier nicht berücksichtigt. Für 
unsere Zwecke reicht eine Unterscheidung zwischen positiven, neutralen und negativen Einstellungen zu politischen Objekten aus. Das Vorherrschen positiver politischer Einstellungen bezeichneten ALMOND und VERBA (1965: $20 \mathrm{ff}$.) als "allegiance" oder politische Unterstützung, die Dominanz negativer Einstellungen als Entfremdung, das Überwiegen neutraler Einstellungen als Apathie. In stabilen politischen Systemen, insbesondere in stabilen Demokratien, überwiegen ihrer Auffassung nach im allgemeinen die positiven Einstellungen, d.h. die Politische Kultur entspricht der politischen Struktur (ALMOND/VERBA 1965: 31 ff.) Dabei blieb offen, wie verbreitet die positiven Einstellungen sein müssen und ob alle politischen Objekte auf das gleiche Ausmaß an Unterstützung angewiesen sind.

Eine große Bedeutung für die Typologisierung und empirische Erforschung Politischer Kulturen kommt der Verteilung der Einstellungen zu einzelnen politischen $\mathrm{Ob}$ jekten zu. In Anlehnung an EASTONs (1965) Systemmodell der Politik gliederten AL MOND/VERBA die Gesamtmenge politischer Objekte in vier Teilklassen auf: das politische System als allgemeines Objekt, das Input- sowie das Output-Subsystem und die Rolle des Bürgers als aktiver Partizipant an der Politik.

Als Inputs bezeichnet man Impulse, die das politische System aus seinen verschiedenen Umwelten, z.B. aus der Gesellschaft, dem Wirtschaftssystem oder anderen Staaten erhält. EASTON rechnet zur Input-Seite der Politik diejenigen Handlungen, Organisationen und Prozesse, durch die das politische System Forderungen aus seiner Umwelt aufnimmt, sie bearbeitet und durch die es sich politische Unterstützung beschafft. Die politischen Parteien, Interessenverbände und Bürgerinitiativen erfüllen derartige Funktionen. Aber auch in Demonstrationen, Unterschriftenaktionen oder Gesprächen einzelner Personen mit Politikern oder Behördenvertretern werden Forderungen vorgetragen oder wird Unterstützung gewährt bzw. entzogen. Die InputStrukturen und die auf sie gerichteten Einstellungen bezeichne ich als die partizipative Komponente der Politik. Sie ist auf die Vorbereitung der Produktion politischer Entscheidungen ausgerichtet (vgl. auch: LEHMAN 1971).

Die Outputs umfassen die Handlungen, Organisationen usw., durch die das politische System materielle oder immaterielle Leistungen an seine Umwelt abgibt, z.B. in Form von Gesetzen, Sozialleistungen, der Unterhaltung von Schulen oder auch von Zeremonien und öffentlichen Erklärungen von Politikern. Als Produzenten politischer Outputs kommen alle Träger autoritativer Entscheidungsfunktionen in Frage, vor allem Parlament, Regierung und Verwaltung. Im Output-Bereich geht es um die Durchsetzung politischer Entscheidungen, also um die regulative Komponente der Politik.

Unklar blieb in den Überlegungen von ALMOND/VERBA das Verhältnis der Inputs und der Outputs zum System als allgemeinen Objekt. Üblicherweise benutzt man den Begriff "System" als Bezeichnung für die Summe seiner Teile und der zwischen ihnen bestehenden Beziehungen. EASTON (1965) konkretisiert den abstrakten Begriff des politischen Systems und unterscheidet zwischen dem politischen Regime und der politischen Gemeinschaft als Orientierungsobjekten. Das politische Regime umfaßt sämtliche politischen Werte, Normen und Autoritätsstrukturen, die Symbole des politischen Kollektivs sowie die zwischen diesen Elementen bestehenden Beziehungen. Die Alltagssprache und die traditionelle Politikwissenschaft bezeichnen diese Sachverhalte als verfassungsmäßige Ordnung. $\mathrm{Zu}$ den typischen Merkmalen liberal-demokratischer Regime gehören unter anderem die Garantie von Grundrechten, die Volkssouveränität und die Gewaltenteilung. Den Begriff politische Gemeinschaft verwen- 
det EASTON zur Bezeichnung der Einheit, der sich Individuen zugehörig fühlen, z.B. zur Nation oder zur Gemeinde.

Die Einführung des Bewertungsobjektes "der Bürger als aktiver Partizipant" soll der Tatsache Rechnung tragen, daß die Politische Kultur nicht nur die Einstellungen zur politischen Umwelt, sondern auch die zur eigenen Rolle in der Politik umfaBt. Diese prinzipiell sinnvolle Überlegung führten ALMOND und VERBA aber nicht konsequent zu Ende. Aus der komplexen Rolle des Staatsbürgers griffen sie lediglich einen Teilaspekt heraus, die zum Input-Bereich gehörige Partizipantenrolle. Andere wichtige Aspekte des Bürgerstatus, wie die Position als Mitglied der politischen Gemeinschaft oder als Adressat staatlicher Leistungen berücksichtigten sie nicht. Wenn man aber schon zwischen Einstellungen zur eigenen Rolle und zur Umwelt unterscheidet, dann sollte man dies für alle Klassen politischer Objekte tun. Da die Trennung zwischen Selbst- und Umweltkomponente für die Typologie Politischer Kulturen keine Rolle spielt, bleibt sie hier außer Betracht. Eine systematisch vollständige Analyse der Politischen Kultur erfaßt somit die Einstellungen der Bevölkerung zum politischen Regime, zur politischen Gemeinschaft sowie zu den partizipativen und regulativen Aspekten der Politik. Diese Orientierungen können positiv, neutral oder negativ ausgeprägt sein (vgl. Abbildung 1).

Abbildung 1: Matrix zur Beschreibung Politischer Kulturen

\begin{tabular}{|l|l|l|l|}
\hline & \multicolumn{3}{|c|}{ Orientierungsart: } \\
Orientierungsobjekt: & positiv & neutral & negativ \\
\hline Politische Gemeinschaft & & & \\
\hline Politisches Regime & & & \\
\hline Partizipative Aspekte der Politik & & & \\
\hline Regulative Aspekte der Politik & & & \\
\hline
\end{tabular}

\subsubsection{Die Verteilung der Orientierungen:}

Homogene und gespaltene Politische Kulturen

Bereits aus der Definition Politischer Kultur als Verteilung individueller Orientierungen auf politische Objekte folgt, daß nicht alle Mitglieder eines Kollektivs die gleichen politischen Einstellungen aufweisen. $\mathrm{Zu}$ den wichtigsten Unterscheidungsmerkmalen Politischer Kulturen gehört demnach der Grad ihrer Homogenität bzw. Heterogenität. Diese Unterscheidung findet sich bereits in der ersten Arbeit über das Konzept der Politischen Kultur. In homogenen Politischen Kulturen, die ALMOND (1956) in den anglo-amerikanischen Demokratien verwirklicht sah, besteht eine breite Übereinstimmung über die Grundlagen des Zusammenlebens in der politischen Gemeinschaft und über die für den Ablauf politischer Prozesse maßgeblichen Spielregeln, also ein Konsens in den Einstellungen zum politischen Regime und zur politischen Gemeinschaft. Als Prototypen von Staaten mit fragmentierten oder gespaltenen 
Politischen Kulturen schätzte ALMOND Italien, Frankreich und Deutschland während der Weimarer Republik ein, deren Bürgerinnen und Bürger in den wichtigsten Fragen des politischen Zusammenlebens unterschiedliche Überzeugungen vertraten. Zudem variierten diese Einstellungsunterschiede systematisch mit der Zugehörigkeit zu bestimmten gesellschaftlichen Gruppen, z.B. mit der Konfessions- oder der Schichtzugehörigkeit, der Parteibindung oder der regionalen Herkunft. An Stelle der nationalen Gemeinschaft und des nationalen Regimes bildeten die Institutionen, Werte und Symbole der jeweiligen Teilgruppe den Kristallisationskern der politischen Loyalität der Bevölkerung. Unter diesen Bedingungen entsteht keine einheitliche nationale Politische Kultur. Statt dessen entwickeln sich mehrere politische Subkulturen mit eigenständigen und zum Teil sogar gegensätzlichen politischen Einstellungen. Es liegt auf der Hand, daß das für Länder mit fragmentierten Politischen Kulturen charakteristische Fehlen gemeinsamer politischer Grundüberzeugungen die Funktionsfähigkeit des politischen Systems beeinträchtigt. Dies gilt insbesondere für Demokratien, zu deren Bestands- und Funktionsvoraussetzungen ein Konsens über bestimmte Grundwerte und Spielregeln gehört.

\subsubsection{Die Staatsbürgerkultur als Politische Kultur der Demokratie}

Trotz der großen Bedeutung, die man der Politischen Kultur als Bestimmungsfaktor der Systemstabilität zuschreibt, liegen über die Merkmale einer demokratischen Politischen Kultur merkwürdigerweise nur ziemlich unbestimmte Aussagen vor. ALMOND/VERBA (1965: 29 ff., 337 ff.) beschrieben die "Staatsbürgerkultur" als die der Demokratie angemessene Politische Kultur nur allgemein und ungenau. In der Politischen Kultur der Demokratie verfügt ein gewisser, aber nicht genau festgelegter Teil der Bevölkerung über politische Grundkenntnisse, er weist eine positive gefühlsmäßige Bindung an die Input- und Output-Strukturen bzw. -Prozesse sowie an das Gesamtsystem auf. Da es sich bei der Staatsbürgerkultur um eine "gemischte Politische Kultur" handelt, müssen nicht alle politischen Akteure zu jeder Zeit eine positive Beziehung zu allen politischen Objekten aufweisen. Auf der anderen Seite darf aber auch keine starke und dauerhafte Spaltung in Subkulturen auftreten, die einzelne Bevölkerungsgruppen von den Institutionen und Symbolen des nationalen politischen Systems entfremdet. Welche konkrete Verteilung von Einstellungen einer demokratischen Politischen Kultur entspricht, ließen ALMOND und VERBA offen. Allenfalls aus ihrer Charakterisierung der Politischen Kultur der Vereinigten Staaten und Großbritanniens als Staatsbürgerkultur sowie aus ihrer kritischen Beschreibung der deutschen Untertanenkultur bzw. der entfremdeten Politischen Kultur Italiens ergeben sich gewisse Hinweise auf das für eine funktionierende Demokratie erforderliche Mindestma $ß$ an Kenntnissen, politischem Kompetenzbewußtsein, politischer Unterstützung usw. Generell sollte die Unterstützung des politischen Regimes und der politischen Gemeinschaft möglichst breit ausfallen. Auf die Input- und die OutputAspekte der Politik sollten sich zumindest auf Dauer nicht mehr negative als positive oder neutrale Einstellungen richten.

Im Gegensatz zu den eingangs erwähnten normativen Konzepten Politischer Kultur verzichteten ALMOND/VERBA auf eine ausdrückliche demokratietheoretische Untermauerung ihres Ansatzes. Sie lieferten zwar ein Kategoriensystem, mit dessen Hilfe man Politische Kulturen beschreiben kann, aber keinen präzisen und überpriufbaren Kriterienkatalog, an dem sich die demokratische Qualität einer Politischen Kul- 
tur festmachen lieBe (vgl. zur Typologisierung Politischer Kulturen auch DIAS 1971; GABRIEL 1986: 62 ff.).

Die folgende Untersuchung kann demnach nicht von einer eindeutig definierten Konzeption kommunaler Demokratie und einer ihr entsprechenden Politischen Kultur ausgehen. Im Hinblick auf die eingangs formulierten Ziele der empirischen Forschung ist dies allerdings auch nicht als Nachteil anzusehen; denn unser Interesse richtet sich ausschließlich darauf, die Einstellungen der Bevölkerung zur lokalen Politik zu beschreiben und zu erklären. Dies setzt voraus, daß zunächst erläutert wird, wodurch sich die lokale und die nationale Politische Kultur voneinander unterscheiden.

\section{Übungsaufgabe 1:}

Erläutern Sie das vom Autor gewählte Konzept Politischer Kultur! Grenzen Sie es vom Alltagsverständnis und von anderen wissenschaftichen Varianten des Begriffes $a b !$

\section{Lokale Politische Kultur}

\subsection{Forschungsrichtungen, Datenlage und methodisches Vorgehen}

Was für die Erforschung Politischer Kultur im allgemeinen festgestellt wurde, gilt auch für die Erforschung lokaler Politischer Kultur: Die Sache ist wesentlich älter als der Begriff. Obgleich die kommunalwissenschaftliche Literatur einige Aussagen über die Einstellungen der Bürgerinnen und Bürger zur Kommunalpolitik enthält, findet der Begriff "lokale Politische Kultur" jedoch nur selten Verwendung (Ausnahmen sind z.B.: BIEGE et al. 1978; GREIFFENHAGEN 1987; JARREN et al. 1989; MIELKE/ OBERNDÖRFER/SCHMITT 1990; SCHACHT 1987).

In theoretischer Hinsicht ergeben sich bei der Anwendung des Konzeptes der Politischen Kultur in der lokalen Politikforschung keine grundsätzlich neuen Probleme. Die politischen Institutionen der Gemeinden gehören zum politischen System eines Staates, sie sind also mögliche Objekte politischer Orientierungen. Im Bezugsrahmen des engeren psychologischen Ansatzes befaßt sich die Erforschung lokaler Politischer Kultur demnach mit einem Ausschnitt aus der umfassenderen Politischen Kultur eines Landes, nämlich mit der Beschreibung und Erklärung gemeindetypischer Einstellungen zur Politik. Dem Attribut "gemeindetypisch" kommt dabei eine Doppelbedeutung zu. Auf der einen Seite stellen Kommunen, ihre Institutionen und die in ihnen ablaufenden Prozesse Objekte dar, die die Bevölkerung - ähnlich wie die nationale Politik wahrnimmt und bewertet. Andererseits bezeichnet der Begriff "lokale Politische Kulturen" auch die für die Bevölkerung einer bestimmten Gemeinde charakteristischen Einstellungen zur Politik. Auch diese Begriffsverwendung entspricht der des Begriffs "nationale Politische Kultur", der zur Abgrenzung einer Nation von anderen Nationen dient.

Im Gegensatz zur Übertragung des theoretischen Konzeptes auf die kommunale Politik stellt der überaus dürftige Stand der empirischen Forschung auf diesem Gebiete die folgende Untersuchung vor erhebliche Probleme. Abgesehen vom Fehlen empirischer Analysen der Beziehungen der Bürgerinnen und Bürger zur Gemeinde- 
politik stehen selbst unausgewertete Daten nur in begrenztem Umfange für Sekundäranalysen zur Verfügung. Aus diesem Grunde ist es schwierig, ein verläBliches Bild der lokalen Politischen Kultur der Bundesrepublik zu zeichnen. Aussagen über ihre Eigenschaft stiitzen sich hauptsächlich auf vier Arten von Quellen mit sehr unterschiedlichem Informationswert.

Die ersten gemeindesoziologischen Einzelfallstudien, die politikwissenschaftliche Fragen behandeln, stammen bereits aus den fünfziger Jahren. Seither wurden zwar etliche empirische Gemeindestudien publiziert, wie die frühen Arbeiten enthalten sie aber in der Regel keine interkommunal vergleichbaren Daten (vgl. die zusammenfassenden Übersichten bei GABRIEL 1979: 107 ff.). Neuere, von mehreren Stadtverwaltungen in der Bundesrepublik durchgeführte Bevölkerungsumfragen informieren den Leser nur über Teilaspekte der lokalen Politischen Kultur. Im Vordergrund steht zumeist die Zufriedenheit der Bevölkerung mit dem Infrastrukturangebot in der betreffenden Stadt und ihre Präferenz für bestimmte kommunale Leistungen. Die in diesen Studien enthaltenen Daten sind nur zum Teil miteinander vergleichbar. Darüber hinaus lassen sie grundlegendere Aspekte der lokalen Politischen Kultur, z.B. die Einstellung zu den lokalen politischen Institutionen und Prozessen unberücksichtigt (vgl. die Übersicht bei BRETSCHNEIDER/BICK 1989a; 1989b). Aus diesen Gründen erscheint mir ihr theoretischer Ertrag für die Erforschung lokaler Politischer Kultur begrenzt, unabhängig von ihrem Nutzen für die Beschreibung der politischen Verhältnisse in einzelnen Städten. Über die für eine Gemeinde typische Politische Kultur geben sie keine Aufschlüsse, weil derartige Aussagen nur in vergleichender Perspektive möglich sind.

Vergleichende Städtestudien auf dem Gebiet der Einstellungs- oder Kulturforschung stellen nach wie vor eine Mangelware dar. Die bislang vorgelegten Arbeiten schließen ausnahmslos nur wenige Kommumen ein (vgl. ARZBERGER 1980; ARZBERGER/MURCK/SCHUMACHER 1979; HILL 1987). Etwas breiter angelegte Untersuchungen der politischen Einstellungen von Spezialgruppen, z.B. von Ratsmitgliedern oder Bürgermeistern (GABRIEL/HAUNGS/ZENDER 1984; GRAUHAN 1970; KÖSER/CASPERSMerck 1989; SIMON 1988; WEHLING/SIEWERT 1987), informieren lediglich über die politischen Orientierungen der Inhaber kommunaler Führungspositionen, aber nicht über die Einstellungen der Bevölkerung. Ob und wieweit die Einstellungen der $\mathrm{Be}$ völkerung und der lokalen Führungsgruppen miteinander übereinstimmen, ist eine außerordentlich interessante, aber nur durch empirische Analysen zu klärende Frage (vgl. hierzu ARZBERGER 1980). Das durch die vorliegenden Studien vermittelte Bild der lokalen Politischen Kultur weist große Lücken auf. Vor allem läßt es keine Aussage darüber zu, wie typisch oder atypisch die Verhältnisse in einzelnen Städten für die lokale Politische Kultur der Bundesrepublik sind.

Eine dritte Forschungsstrategie besteht darin, Informationen über kommunalpolitische Einstellungen aus nationalen Repräsentativerhebungen zu gewinnen (vgl. z.B. OEL 1972; PAPPI 1970; GABRIEL 1990a; 1991). Im Vordergrund stehen Einzelprobleme, eine umfassende Charakterisierung der lokalen Politischen Kultur der Bundesrepublik scheitert an der Datenlage. Zudem enthalten diese Untersuchungen keine Informationen über die Einstellungen der Bevölkerung einzelner Gemeinden. Auf Grund der kleinen Zahl von Befragungen in jeder Gemeinde können sie auch nicht nachträglich durch Spezialauswertungen gewonnen werden.

Daneben gibt es schließlich Aussagen über lokale Politische Kulturen, die auf Erfahrungsberichten (vgl. z.B. SCHNEIDER 1979) oder auf Spekulationen über die kom- 
munalpolitischen Einstellungen der Bevölkerung und über ihre Besonderheiten gegenüber der Bundespolitik beruhen (vgl. GRAUHAN 1971; HALLER 1979; LEHM. BRUCH 1979; vgl. zur Zusammenfassung den Überblick bei GABRIEL 1979). Derartige Studien sind zwar für die Formulierung von Hypothesen über die Eigenschaften der lokalen Politischen Kultur nützlich, empirisch überprüfte Erkenntnisse dagegen enthalten sie nicht.

\subsection{Verwendungsweisen des Begriffs lokale Politische Kultur}

\subsubsection{Lokale Politische Kultur als System von Einstellungen zur lokalen Politik}

Auf der Grundlage des psychologischen Ansatzes ist als lokale Politische Kultur zunächst die Verteilung der Einstellungen der Bevölkerung zur Gemeinde und zu ihren politischen Strukturen und Prozessen zu verstehen. Da sich im Laufe der Entwicklung der kommunalen Selbstverwaltung auf der lokalen Ebene im Prinzip ähnliche politische Strukturen und Prozesse herausbildeten wie in der nationalen Politik, läßt sich das für die Beschreibung der nationalen Politik entwickelte Kategoriensystem auch für die Analyse der lokalen Politischen Kultur verwenden. Im Rahmen der Binnengliederung des nationalen politischen Systems nimmt die Bürgerschaft das lokale politische System als eine Einheit mit eigenen Aufgaben, Institutionen und Prozessen wahr und setzt sich mit diesen Objekten auseinander. Neben dem gesamten politischen System der Gemeinde sind die einzelnen Träger kommunalpolitischer Entscheidungskompetenzen und die Teilnehmer am politischen WillensbildungsprozeB der Gemeinde mit den politischen Erwartungen der Gemeindebewohner konfrontiert. Diese richten sich auf den Bürgermeister, die Kommunalvertretung und die Kommunalverwaltung, die lokalen Interessengruppen, die politischen Parteien und die lokalen Massenmedien. Kommunalwahlen, Bürgerversammlungen, Bürgerbegehren, Bürgerentscheide und zahlreiche weniger formalisierte Prozesse dienen dem Austausch zwischen den lokalpolitischen Entscheidungsträgern und der Bevölkerung. Auch wenn die Gemeindeordnungen der Bundesländer diese Akteure, Strukturen und Prozesse unterschiedlich bezeichnen und wenn in ihrem praktischen Funktionieren gewisse gemeindespezifische Besonderheiten erkennbar sind, weisen die Kommunen der Bundesrepublik ein gemeinsames Merkmal auf: Die Bürger erwarten von den kommunalen Institutionen und Akteuren die Erfüllung bestimmter Aufgaben. Auf diese Forderungen reagieren die Inhaber lokalpolitischer Entscheidungspositionen mit materiellen, regulativen und symbolischen Leistungen. Die genaue Beschaffenheit der kommunalpolitischen Einstellungen und das sich daraus ergebende Muster lokaler Politischer Kultur läßt sich nur empirisch ermitteln (vgl. zur Einführung in die Strukturen und Prozesse des lokalen politischen Systems z.B. GABRIEL 1979; 1989; GUNLICKS 1986; NASSMACHER/NASSMACHER 1979).

Somit bezeichnet der Begriff "lokale Politische Kultur" die in der Bevölkerung vorhandenen Einstellungen zur Kommunalpolitik allgemein und zu ihren einzelnen Aspekten. $\mathrm{Zu}$ ihr gehören unter anderem das kommunalpolitische Wissen, das Interesse an der Kommunalpolitik, die Identifikation mit der Gemeinde, die Einschätzung kommunalpolitischer Beteiligungsmöglichkeiten, die kommunalpolitischen Prioritäten und die Zufriedenheit mit dem kommunalen Leistungsangebot sowie die Einstellung zu den Kommunalparteien, zur Kommunalvertretung und zur Kommunalverwaltung. Die Verteilung dieser Einstellungen unter den Bundesbürgern konstituiert 
die lokale Politische Kultur der Bundesrepublik. Dieser bisher vernachlässigte Aspekt der Analyse lokaler Politischer Kulturen steht im Mittelpunkt dieses Beitrages. Im Rahmen der durch die Datenlage gegebenen Möglichkeiten werden die Einstellungen der Bevölkerung zur Gemeinde und ihren politischen Merkmalen untersucht und vergleichbaren Einstellungen zur nationalen Politik gegenübergestellt.

\subsubsection{Lokale Zugehörigkeit als Erklärungsfaktor Politischer Kultur}

Eine zweite Variante des Begriffs lokale Politische Kultur beruht auf der Annahme, da $B$ sich in einzelnen Gemeinden besondere politische Traditionen und Orientierungsmuster herausbilden, durch die sie sich von anderen Kommunen unterscheiden. Während die lokale Politische Kultur in der ersten Bedeutungsvariante über die Gemeinde als politisches Objekt definiert wurde, stehen in der zweiten Konzeption die gemeindespezifischen Ausprägungen der Einstellungen zur lokalen und nationalen Politik im Vordergrund (vgl. WEHLING 1987: $261 \mathrm{ff}$.).

Die Vorstellung, daß einzelne Kommunen oder Gemeindetypen die Grundlage für die Ausbildung spezieller lokaler Subkulturen darstellen, hat in der Erforschung Politischer Kultur eine lange Tradition. ALMOND/VERBA (1965: 16 f., 26 ff.; vgl. auch INRELES 1974; VERBA 1978) stellen die "parochiale" Politische Kultur oder die "Kirchtumskultur" als einen eigenständigen Kulturtyp dar. Zu ihren wichtigsten Eigenschaften gehört die Begrenzung der politischen Vorstellungswelt der Bevölkerung auf die engere Gemeinde: Sie räumt der nationalen Politik keine Bedeutung für ihr Leben ein und verfügt nicht über differenzierte Kenntnisse der nationalen Politik. Loyalitätsbeziehungen zum nationalen politischen System, seinen Institutionen und Symbolen kommen nicht zustande. Diese gemeindezentrierten Politischen Kulturen waren für traditionale Gesellschaften mit voneinander isolierten Gemeinden, geringer Arbeitsteilung, fehlender interkommunaler Mobilität, wenig entwickelten nationenweiten Kommunikationssystemen und einer Konzentration aller lebenswichtigen menschlichen Aktivitäten auf die Wohngemeinde typisch.

In modernen Gesellschaften sind die technologischen und ökonomischen Grundlagen für die Ausbildung parochialer Kulturen entfallen (vgl. auch SCHNEIDER 1979: 18 ff.). Die Einstellungen der Bevölkerung zur lokalen und zur nationalen Politik haben sich einander angeglichen und werden von denselben Faktoren bestimmt. Dennoch können sich im Zusammenleben in einer Kommune weiterhin besondere politische Einstellungen entwickeln, durch die sie sich von den Kommunen in ihrer Umgebung unterscheidet. Der Versuch, derartige Besonderheiten zu beschreiben, scheitert in der Forschungspraxis allerdings häufig an der Datenlage. Die einstellungsprägenden politischen Ereignisse liegen oft in der Vergangenheit, empirische Daten über ihren Ablauf und über ihren Einfluß auf das Entstehen politischer Einstellungen liegen im Regelfalle nicht vor.

Als ein weiteres schwieriges Problem erweist sich das Verhältnis von Politischer Kultur und Sozialstruktur. Zwar ist es plausibel, auf Besonderheiten der Politischen Kultur von Berlin-Kreuzberg, Hamburg-Blankenese, Passau oder Rüsselsheim hinzuweisen, doch übersieht man dabei leicht, $\mathrm{da}$ B die Unterschiede zwischen diesen Kommunen sich möglicherweise aus der Sozialstruktur ergeben und keine eigenständigen kulturellen Größen widerspiegeln. Das Problem, daß vermeintliche kulturelle Besonderheiten bei einer Kontrolle sozialstruktureller Faktoren verschwinden oder doch zumindest an Bedeutung verlieren, wird in den vorliegenden Untersuchungen 
lokaler Politischer Kulturen nicht einmal angesprochen, geschweige denn einer Lösung nähergebracht.

Wesentlich einfacher läßt sich ein letztes mit der Untersuchung lokaler Politischer Kulturen verbundenes Problem klären, nämlich die Frage, ob deren Ausprägungen systematisch vom Gemeindetypus beeinflußt werden. In diesem Bezugsrahmen stand beispielsweise die Untersuchung von PAPPI (1970) zur Bedeutung des Stadt-LandUnterschiedes für die Politische Kultur der Bundesrepublik. Eine derartige Fragestellung ist theoretisch-systematisch sinnvoll. Die Gemeindesoziologie geht von der Annahme aus, daß sich städtische und ländliche Lebensformen voneinander unterscheiden. Insofern weisen Städte und ländliche Gebiete möglicherweise besondere Ausprägungen einer Politischen Kultur auf. Die Frage, ob sich die politischen Einstellungen der Stadt- und der Landbevölkerung voneinander unterscheiden, läßt sich bei der gegebenen Datenlage vergleichsweise einfach klären, da die meisten nationenweiten Umfragen Angaben über die Wohnortgröße oder den Gemeindetyp enthalten. In diesem Sinne werde ich in meiner Analyse der lokalen Politischen Kultur der Bundesrepublik die Gemeindegröße als ein mögliches Unterscheidungsmerkmal behandeln.

\section{Geschichtliche Rahmenbedingungen der lokalen Politischen Kultur in der Bundesrepublik Deutschland}

Für das Verständnis der Eigenschaften der lokalen Politischen Kultur der Bundesrepublik ist ein kurzer Rückblick auf die Entwicklung der kommunalen Selbstverwaltung in Deutschland nützlich, weil sich die Einstellungen der Bevölkerung zur Kommunalpolitik in einem längeren ProzeB herausbilden. Einerseits stehen diese Orientierungen in der Kontinuität einer bestimmten Selbstverwaltungstradition, andererseits reflektieren sie den verfassungspolitischen Neubeginn nach 1945.

Vermutlich spielten die Gemeinden in der Vorstellungswelt der Bürger bereits frühzeitig eine wichtige Rolle. Die Tradition städtischer Selbstverwaltung reicht in Deutschland bis ins Mittelalter zurück. In Preußen markieren die Steinschen Reformen (1808) den Beginn einer modernen kommunalen Selbstverwaltung. Im Zuge dieser Reformen erfolgte eine weitgehende Dezentralisierung der staatlichen Aufgaben. Dabei erhielten die Kommunen das Recht, besonders bürgernahe Angelegenheiten eigenverantwortlich zu erledigen sowie eine gewisse Autonomie in der Gestaltung ihrer Einnahmen und Ausgaben. Die Zuständigkeit für die Erfüllung der kommunalen Aufgaben lag bei einer direkt von der Bevölkerung gewählten Gemeindevertretung und einem durch diese bestellten Magistrat. In den meisten anderen deutschen Staaten erhielten die Städte im Laufe des 19. Jahrhunderts gleichfalls Selbstverwaltungsrechte. Auch wenn die Verfassung der Kommunen von unseren heutigen Demokratievorstellungen noch relativ weit entfernt war und obgleich die Demokratisierung der Gemeindepolitik immer wieder von Rückschlägen unterbrochen wurde, kam der frühzeitigen Institutionalisierung kommunaler Selbstverwaltungsrechte vermutlich eine große Bedeutung für die Beziehung der Bevölkerung zu ihrer Gemeinde zu: Zumindest das Besitzbürgertum verfügte frühzeitig über politische Mitwirkungsrechte, aus denen sich eine enge Bindung an die Gemeinde und ihre politischen Institutionen und Prozesse entwickeln konnte. Selbst der gröBere Teil der Bevölkerung, dessen Mitwir- 
kungsrechte stark begrenzt waren, dürfte das lokale politische System als eine eigenständige Handlungseinheit wahrgenommen haben, der man sich zugehörig fühlte und die Leistungen erbrachte (zur Entwicklung der kommunalen Selbstverwaltung in Deutschland vgl. z.B. den Überblick bei GuNLICKs 1986: 5 ff.).

Durch die Institutionalisierung politischer Mitwirkungsrechte in den Städten kam der kommunalen Selbstverwaltung zwar eine Vorreiterfunktion für die Entwicklung der Demokratie in Deutschland zu. Auf der anderen Seite blieb ihr Beitrag zur Demokratisierung Deutschlands jedoch eher begrenzt; denn auf der kommunalen Ebene bot sich der Bevölkerung vor der Gründung der Bundesrepublik allenfalls bedingt eine Chance, demokratische Werte, Normen und Verhaltensweisen zu erlernen. Ein wichtiger Grund für die demokratietheoretische Ambivalenz der deutschen Selbstverwaltungstradition liegt in der vorherrschenden Interpretation der Beziehung zwischen den Gemeinden und dem Staat. Im 19. und frühen 20. Jahrhundert sah man die Kommunen nicht als integrierte Bestandteile des Staates, sondern als aus dem Staat ausgegrenzte und ihm gegenüber mit besonderen Rechten ausgestattete Lebensbereiche an. Im autoritären Obrigkeitsstaat Preußens erfüllte die Institutionalisierung kommunaler Selbstverwaltungsrechte nicht zuletzt die Funktion, weitergehende, auf den Staat gerichtete Demokratisierungsfordenungen abzuwehren. Die Staatsrechtslehre untermauerte diese Strategie durch die Behandlung des States und der Gemeinden als wesensmäßig voneinander verschiedene Daseinsbereiche. Bürgerschaftliche Selbstverwaltung konnte zwar in genossenschaftlich-gesellschaftlichen Lebensbereichen praktiziert werden, die Leitung des Staates aber unterlag anderen Ordnungsprinzipien (vgl. ZIPFEL 1979: 139).

Trotz der Demokratisierung des Kommunalwahlrechts blieb die Antinomie zwischen Staat und Gemeinde auch für das Verfassungsdenken der Weimarer Republik bestimmend. Im Gegensatz zum Grundgesetz, in dem das Selbstverwaltungsrecht der Kommunen in den Abschnitt über die Beziehungen zwischen dem Bund und den Ländern eingeordnet ist, finden sich die auf die kommunale Selbstverwaltung bezogenen Bestimmungen der Weimarer Reichsverfassung im Grundrechtsteil. Hierin zeigt sich die verfassungspolitische Kontinuität der traditionellen Selbstverwaltungsdoktrin: Wie im Kaiserreich blieb die kommunale Selbstverwaltung eine vorstaatliche, gesellschaftliche Einrichtung.

Erst im Zuge der Re-Education-Bemühungen der Alliierten nach dem Zweiten Weltkrieg erfolgte eine politische Neubestimmung der kommunalen Selbstverwaltung. Neben ihren traditionellen administrativen Zuständigkeiten für die wirtschaftliche, soziale und kulturelle Daseinsvorsorge wurde den Kommunen nunmehr die politische Aufgabe zugewiesen, zwischen dem Bürger und dem Staat zu vermitteln und den Aufbau einer funktionsfähigen Demokratie von der Basis her zu fördern (vgl. STAMMEN 1977). In diesem Sinne integrierte das Grundgesetz die Kommunen in den demokratischen Staat. Es bleibt aber zu prüfen, ob die Bevölkerung und die politischen Führungsgruppen diese Neudefinition der kommunalen Selbstverwaltung mitvollzogen und ob damit der traditionelle Gegensatz zwischen Staat und Gemeinden überwunden wurde.

Aus der politischen Entwicklung Deutschlands im 19. und 20. Jahrhundert ergibt sich somit ein Spannungsverhältnis zwischen dem Staat und der kommunalen Selbstverwaltung. Nach der Demokratisierung des politischen Institutionensystems und mit dem schrittweisen Aufbau einer demokratischen Politischen Kultur in der Bundesrepublik (vgl. BAKER/DALTON/HILDEBRANDT 1981; CONRADT 1980; GABRIEL 1986) 
stellt sich demzufolge die Frage, ob die Bevölkerung unter den Bedingungen einer pluralistischen Demokratie noch an der traditionellen Gegenüberstellung von Staat und Selbstverwaltung festhält und an welchen konkreten Eigenschaften der lokalen und nationalen Politik sich dies zeigt. In mehreren Arbeiten finden sich Hinweise auf die weite Verbreitung der traditionellen, "vordemokratischen" Konzeption kommunaler Selbstverwaltung. Als Charakteristika der lokalen Politischen Kultur, insbesondere kleiner Gemeinden, stellt LEHMBRUCH (1979: 324 ff.) die folgenden Merkmale heraus: Die kommunalpolitischen Vorstellungen der Bürgerinnen und Bürger seien "unpolitisch". Die Bevölkerung betrachte die kommunale Politik nicht als einen eigenständigen, aus dem übrigen sozialen Leben der Gemeinden ausdifferenzierten Handlungsbereich. In der Tätigkeit des Bürgermeisters als der exponierten lokalen Führungsfigur überwiege die fachlich-administrative Komponente, Parteien würden als Fremdkörper in der Kommunalpolitik eingeschätzt, politische Probleme sollten möglichst einvernehmlich geregelt werden. Andererseits sei das politische KompetenzbewuBtsein der Bürger in lokalen Fragen deutlich stärker ausgeprägt als in den Angelegenheiten der nationalen Politik (ähnlich für Großstädte: GRAUHAN 1971: 107 ff.). Es wird in den folgenden Teilen dieses Beitrages zu prüfen sein, ob diese Annahmen über die Eigenschaften der lokalen Politischen Kultur der Bundesrepublik der Wirklichkeit entsprechen, ob sie ausschließlich für die lokale Politische Kultur typisch sind oder sich auch in den Einstellungen zur nationalen Politik finden und ob sich die lokale Politische Kultur der Bundesrepublik als eine Einheit darstellt oder ob sie in mehrere Subkulturen zerfällt, insbesondere in eine städtische und eine ländliche Politische Kultur.

\section{Die lokale Politische Kultur der Bundesrepublik Deutschland}

Eine auch nur annähernd vollständige Beschreibung der lokalen Politischen Kultur der Bundesrepublik ist in Anbetracht der außerordentlich lückenhaften Datenbestände nicht möglich. Vielmehr muß sich die folgende Untersuchung darauf beschränken, beispielhaft einige charakteristische Züge im Verhältnis der Bundesbürger zur Kommunalpolitik darzustellen. Selbst dieses eingeschränkte Ziel läßt sich unterschiedlich gut verwirklichen. Dies gilt insbesondere im Hinblick auf die Aktualität der Daten und die Möglichkeit, längerfristige Veränderungen aufzuzeigen. So bleibt das nachfolgend gezeichnete Bild der lokalen Politischen Kultur notwendigerweise bruchstückhaft. Dennoch liegen für einige charakteristische Aspekte unseres Gegenstandes Informationen vor, aus deren Synthese sich eine einigermaßen instruktive Beschreibung der Beziehungen der Bevölkerung zu den Institutionen und Trägern der kommunalen Selbstverwaltung in der Bundesrepublik ergibt.

\subsection{Die allgemeine Beziehung der Bevölkerung zur Gemeinde und zur Kommunalpolitik}

\subsubsection{Die Gemeindeidentifikation}

Als ein grundlegendes Merkmal der Politischen Kultur muß man die Verbundenheit der Bürger mit der politischen Gemeinschaft, der sie angehören, einstufen. Der $\mathrm{Zu}$ - 
sammenhalt einer sozialen oder politischen Gemeinschaft hängt maßgeblich von der Loyalität ihrer Mitglieder ab. Aus vielen Gründen sind derartige Bindungen in modemen pluralistischen Gesellschaften keineswegs selbstverständlich.

Angeblich gehörte die Gemeinde in vormodernen Gesellschaften zu den wichtigsten Identifikationssymbolen. Die Gemeindebindung der Bevölkerung war stärker als die Loyalitätsbeziehung zum Nationalstaat. Dementsprechend sieht man in der Ablösung parochialer Bindungen durch die Loyalität zur nationalen Gemeinschaft einen wesentlichen Aspekt des politischen Modernisierungsprozesses (vgl. z.B. INKELES 1974; VERBA 1978). Die Abschwächung der Identifikation mit der Gemeinde führt man auf deren veränderte Funktion für ihre Bewohner zurück. Moderne, arbeitsteilige Gesellschaften zeichnen sich unter anderem durch eine hohe räumliche Mobilität aus, die nicht zuletzt durch die Trennung von Wohnort, Arbeitsplatz und Ort der Freizeitgestaltung bedingt ist. Unter diesen Bedingungen fungiert der Wohnort nicht mehr als geschlossene soziale Einheit, sondern er ist mit seinem Umland auf vielfältige Weise verflochten. Mit dieser Funktionseinbuße verliert die Wohngemeinde zugleich einen Teil ihrer Integrationsfähigkeit.

Obgleich Gemeindebindungen in modernen Gesellschaften nicht völlig bedeutungslos werden, schwächt sich die Identifikation der Bevölkerung mit ihrer Gemeinde insofern $a b$, als neben sie zahlreiche andere Identifikationsträger treten. Als politische Gemeinschaft tritt die Gemeinde zunehmend hinter den Nationalstaat oder supranationale Zusammenschlüsse zurück. Dies schlägt sich in den politischen Loyalitäten der Bürgerinnen und Bürger nieder. Dennoch bleibt unter diesen veränderten Bedingungen ein Mindestma $\beta$ an Identifikation mit der Wohngemeinde unabdingbar, wenn die Funktionsfähigkeit der kommunalpolitischen Institutionen gesichert werden soll. Die in der Bundesrepublik durchgeführten Verwaltungsreformen verfolgten ausdrücklich das Ziel, die Leistungsfähigkeit der Gemeinden zu erhöhen und gleichzeitig ihre Integrationsfunktion zu erhalten (vgl. hierzu vor allem die klassische Arbeit von WAGENER 1969). Nach HILL (1987: 27 f.) fördert die Gemeindebindung die Anteilnahme am kommunalpolitischen Leben: "Selbst-Verwaltung verlangt zunächst ein 'Selbst'-Bewußtsein der Bürger als Grundlage und Motivation für Selbstverwaltung" (Hervorh. im Original). Insoweit kommt der Gemeindeidentifikation, die im Sinne EASTONs als generelle Unterstützung der lokalen politischen Gemeinschaft zu interpretieren ist, in der lokalpolitischen Kultur eine Schlüsselrolle zu. Dabei schließen lokale und überlokale Bindungen einander keineswegs aus.

Ungeachtet der gesellschaftlich-politischen Umbrüche in der Nachkriegszeit stellte die Gemeinde noch in den siebziger Jahren für die meisten Bundesbürger das wichtigste territoriale Identifikationsobjekt dar. In mehreren einschlägigen Erhebungen über das Gefühl territorialer Zugehörigkeit entfielen die meisten Nennungen auf die Stadt bzw. Gemeinde (1973: 50\%; 1976: 54\%). Mit einem weiten Abstand zur Gemeinde rangierte der Bund an zweiter Stelle (1973: 24\%; 1976: 28\%; vgl. auch Tabelle 1). Wie HILL (1987: $103 \mathrm{ff}$.) im Rahmen seiner Fallstudie in vier Gemeinden ermittelte, schwächte die kommunale Gebietsreform allerdings die Integrationskraft der lokalen Gemeinden nachhaltig. Während vor der Reform 66 Prozent der Befragten die alte Gemeinde als eine Einheit und Gemeinschaft bewerteten, der sie zugehörten und in der sie sich wohlgefühlt hätten, schrieb nur noch ein knappes Viertel (23\%) den neugebildeten Gemeinden diese Eigenschaften zu. Vielfach hielten die Bürgerinnen und Bürger an ihrer Loyalität zum alten Ortsteil fest. Weitere Einzelfallstudien vermitteln ein ähnliches Bild (vgl. JAUCH 1975: 87; ZAHN 1982: 91, 214). 
Tabelle 1: Gemeindeidentifikation in der Bundesrepublik, 1976 und 1990 (Angaben: Prozentanteile)

"Was meinen Sie, welcher der folgenden geographischen Gruppen gehören Sie in erster Linie an?"

Einwohnerzahl der Gemeinde

\begin{tabular}{|c|c|c|c|c|c|c|}
\hline & & $\begin{array}{r}\text { bis } \\
5.000\end{array}$ & $\begin{array}{r}5.000 \\
-19.999\end{array}$ & $\begin{array}{r}20.000 \\
-99.999\end{array}$ & $\begin{array}{c}100.000 \\
\text { u.m. }\end{array}$ & $\begin{array}{r}\text { Alle } \\
\text { Befragten }\end{array}$ \\
\hline Gemeinde & $\begin{array}{l}1976 \\
1990\end{array}$ & $\begin{array}{l}73 \\
38\end{array}$ & $\begin{array}{l}53 \\
32\end{array}$ & $\begin{array}{l}51 \\
34\end{array}$ & $\begin{array}{l}45 \\
30\end{array}$ & $\begin{array}{l}54 \\
33\end{array}$ \\
\hline $\begin{array}{l}\text { Bundes- } \\
\text { republik }\end{array}$ & $\begin{array}{l}1976 \\
1990\end{array}$ & $\begin{array}{r}7 \\
10\end{array}$ & $\begin{array}{l}19 \\
12\end{array}$ & $\begin{array}{l}27 \\
16\end{array}$ & $\begin{array}{l}28 \\
19\end{array}$ & $\begin{array}{l}22 \\
14\end{array}$ \\
\hline $\begin{array}{l}\text { Bundesland } \\
\text { Region }\end{array}$ & $\begin{array}{l}1976 \\
1990\end{array}$ & $\begin{array}{r}6 \\
34\end{array}$ & $\begin{array}{l}11 \\
35\end{array}$ & $\begin{array}{r}8 \\
28\end{array}$ & $\begin{array}{r}8 \\
25\end{array}$ & $\begin{array}{r}8 \\
29\end{array}$ \\
\hline Sonstiges & $\begin{array}{l}1976 \\
1990\end{array}$ & $\begin{array}{l}6 \\
7\end{array}$ & $\begin{array}{r}7 \\
10\end{array}$ & $\begin{array}{l}11 \\
11\end{array}$ & $\begin{array}{l}12 \\
15\end{array}$ & $\begin{array}{r}9 \\
12\end{array}$ \\
\hline k.A. & $\begin{array}{l}1976 \\
1990\end{array}$ & $\begin{array}{r}8 \\
12\end{array}$ & $\begin{array}{l}11 \\
12\end{array}$ & $\begin{array}{r}4 \\
12\end{array}$ & $\begin{array}{r}7 \\
11\end{array}$ & $\begin{array}{r}7 \\
12\end{array}$ \\
\hline $\begin{array}{l}\text { Prozentpunkt } \\
\text { Bund- } \\
\text { Gemeinde }\end{array}$ & $\begin{array}{c}\text { differenz } \\
1976 \\
1990\end{array}$ & $\begin{array}{l}66 \\
28\end{array}$ & $\begin{array}{l}34 \\
20\end{array}$ & $\begin{array}{l}24 \\
18\end{array}$ & $\begin{array}{l}17 \\
11\end{array}$ & $\begin{array}{l}32 \\
19\end{array}$ \\
\hline$N$ & $\begin{array}{l}1976 \\
1990\end{array}$ & $\begin{array}{l}194 \\
287\end{array}$ & $\begin{array}{l}241 \\
566\end{array}$ & $\begin{array}{l}235 \\
578\end{array}$ & $\begin{array}{l}337 \\
371\end{array}$ & $\begin{array}{l}1007 \\
1902\end{array}$ \\
\hline
\end{tabular}

Quelle: Eurobarometer 6, European Values Survey 1990; Auswertung d. Verf.

Diese Auswirkung der Gebietsreform auf die politischen Einstellungen der Bevölkerung erscheint plausibel, wenn man sich den Zusammenhang zwischen der Gemeindegröße und der Gemeindebindung vergegenwärtigt. Mit zunehmender Gemeindegröße wird die Identifikation mit der Gemeinde schwächer, mit der Bundesrepublik jedoch stärker. 1976 nannten $73 \%$ der Befragten aus Gemeinden mit weniger als 5.000 Einwohnem ihren Wohnort, aber nur sieben Prozent die Bundesrepublik als wichtigstes Identifikationsobjekt. In Großstädten mit mehr als 100.000 Einwohnern überwog die lokale Identifikation zwar ebenfalls, jedoch nicht so eindeutig wie in den Kleingemeinden (45 zu 28\%). Die Einstellungen der Klein- und Mittelstädter lagen zwischen diesen Extremen, waren denen der Großstädter aber ähnlicher (vgl. Tabelle 1). Noch deutlicher traten diese Identifikationsmuster bei einem Vergleich der Prozentpunktdifferenz zwischen Gemeinde- und Bundesorientierung in Gemeinden unterschiedlicher Größe hervor: Sie betrug in den kleinen Gemeinden 66, in den Großstädten dagegen nur 17 Punkte. Da im Zuge der Gebietsreform vor allem kleine Gemeinden aufgelöst wurden, nahm die Gemeindebindung der Bevölkerung insgesamt 
ab. Die für "Kirchtumskulturen" typische enge Bindung an die Wohngemeinde ist somit vor allem in kleinen, ländlichen Gemeinden anzutreffen. ${ }^{3}$

Im Vergleich mit 1976, als die Gebietsreform in der Bundesrepublik erst wenige Jahre zurücklag und die alten Identifikationsmuster bei den Bewohnern kleiner Gemeinden offenkundig noch wirksam waren, zeichneten sich die betreffenden Einstellungen im Jahr 1990 durch eine wesentlich gröBere Homogenität aus. Zwar war die Dominanz von Gemeindebindungen in den Kleingemeinden nach wie vor am ausgeprägtesten, jedoch bestanden nur graduelle Unterschiede zwischen den einzelnen Gemeindetypen. Diese Angleichung kam nicht zuletzt durch einen starken Rückgang der Gemeindebindung, insbesondere in den kleinen Kommunen, zustande.

\subsubsection{Das Interesse an der Kommunalpolitik, das subjektive Verständnis kommunaler Fragen und das kommunalpolitische Wissen}

Über die politischen Aspekte der Beziehung der Bevölkerung zu ihrer Gemeinde sagt die Gemeindebindung wenig aus. Informationen hierüber müssen andere, speziell auf die Politik bezogene Einstellungen liefern. Als eine "Basisvariable" im Verhältnis der Bürger zur Politik bezeichnet man in der Literatur die geistige Anteilnahme an politischen Vorgängen oder die politische Involvierung, wie sie sich im politischen Interesse, in der Auseinandersetzung mit politischen Ereignissen oder im Wissen über politische Sachverhalte niederschlägt (vgl. z.B. BAKER/DALTON/ HILDEBRANDT 1981: 51 ff.; CONRADT 1980: 238; MILBRATH/GOEL 1977: 46 ff.).

Für den Ablauf der politischen Prozesse in einer Kommune spielt die politische Involvierung eine wichtige Rolle. Sie entscheidet darüber, ob die Bevölkerung in der Lage ist, politische Ereignisse auf ihre eigene Daseinssphäre zu beziehen, Handlungskompetenz zu entwickeln und die Aktivitäten der politischen Führung kritisch zu begleiten. Nicht zuletzt wegen der engen Bindung vieler Bürger an ihre Gemeinde kann man auch eine Bereitschaft zur Anteilnahme an der Kommunalpolitik unterstellen, zumal die Gemeinde vielfach als ein besonders überschaubarer und bürgernaher Lebensbereich gilt (vgl. HÄTTICH 1977).

Im Gegensatz zum generellen Politikinteresse wurde die Zuwendung der Bevölkerung zu Fragen der lokalen Politik empirisch kaum untersucht. Aus diesem Grunde ist man darauf angewiesen, Aussagen über das lokalpolitische Interesse aus mehreren Einzeluntersuchungen und unveröffentlichten Datenbeständen zusammenzutragen. In einer 1986 durchgeführten Repräsentativerhebung des Sozialwissenschaftlichen Instituts der Konrad-Adenauer-Stiftung gaben fast zwei Drittel der Befragten an, sich gleichermaßen für die Bundes-, die Landes- und die Kommunalpolitik zu interessieren (64\%). Primär bundespolitisch interessiert waren nach eigenem Bekunden 22 Prozent, primär kommunalpolitisch nur acht Prozent. Noch kleiner fiel allerdings der Anteil landespolitisch Interessierter (4\%) aus. Der hohe Anteil allgemein Interessierter stützt die Vermutung, daß die Anteilnahme an kommunalen Fragen die an der nationalen Politik keineswegs ausschließt. Beide Einstellungen sind häufig in einer generellen Disposition verankert (so auch BUSE/NELLES/OPPERMANN 1978: 210). Weniger

3 Neben den Angaben zur Gemeindegröße enthalten die Eurobarometer mit der Unterscheidung zwischen ländlichen Gegenden, Klein- und Mittel- sowie Großstädten eine einfache Gemeindetypologie. Bei einer Verwendung dieser Variablen ergeben sich allerdings keine grundsätzlich neuen Erkenntnisse. 
eindeutig sind in dieser Hinsicht die aus einer älteren Studie OELs (1972: 23 ff.) ableitbaren Schlußfolgerungen. Demzufolge war eine klare Mehrheit der Befragten entweder lokal oder überlokal orientiert. Schränkt man die Aussagen auf die Ausrichtung des politischen Interesses ein, dann dominiert das Interesse an der Bundespolitik (20\%) deutlich gegenüber dem an der Kommunalpolitik (8\%). Nur $11 \%$ gaben an, sich für die Bundes- und die Kommunalpolitik zu interessieren.

Wie aus mehreren Einzelfallstudien hervorgeht, scheint die Anteilnahme der $\mathrm{Be}$ völkerung an der Gemeinde- bzw. Bundespolitik hauptsächlich von den konkreten politischen Bedingungen abzuhängen. So konzentrierte sich das Interesse der Bewohner der fränkischen Rebellengemeinde Ermershausen eindeutig auf lokale Angelegenheiten, bundes- und landespolitischen Vorgängen kam eine ausgesprochen nachrangige Bedeutung zu (vgl. HoltManN/KILLISCH 1989: 32, Tabelle 5). Im Vergleich mit anderen Studien erweist sich dieser Fall jedoch als atypisch. Unter normalen Umständen interessiert sich die Mehrzahl der Bürger für die Kommunal- und die Bundespolitik, auf beide Aktionsebenen des politischen Systems richtet sich ein ungefähr gleich großes Interesse (vgl. BIEGE et al. 1978: 152, Tabelle 30; BUSE/NELLES/OPPERMANN 1978: 209 ff., bes. Tabelle 34; HILL 1987: 210, Tabelle 93; JARREN et al. 1989: 194 ff.). Eine interessante Einzelinformation zur Verteilung des Interesses auf die Träger des lokalpolitischen Entscheidungsprozesses präsentierten BUSE et al. (1978: 209, Tabelle 34): Demnach war das Interesse an der Arbeit der Verwaltung (64\%) und des Rates $(57 \%)$ in Andernach wesentlich stärker entwickelt als an den politischen Parteien (46\%).

Die ersten vergleichenden Informationen über das Verständnis der Bevölkerung für Fragen der lokalen und nationalen Politik erhoben ALMOND und VERBA (1965) im Rahmen der Civic-Culture-Studie (vgl. Tabelle 2). Den Ergebnissen dieser Studie zufolge verfügten die Bundesbürger nach ihrer eigenen Einschätzung bereits im Jahre 1959 über ein gut ausgeprägtes Verständnis der nationalen/internationalen und der lokalen Politik. Dies zeigt sich nicht zuletzt im Vergleich mit etablierten Demokratien. Darüber hinaus stützen die Daten die These von der besonderen Bürgernähe und Überschaubarkeit der Kommunalpolitik. Zwar gab fast jeder zweite Bundesbürger an, die Vorgänge in der nationalen und internationalen Politik gut oder sehr gut zu verstehen, in der lokalen Politik lag der betreffende Anteil mit $62 \%$ aber noch deutlich höher. Lediglich ein Viertel der Befragten verstand nach eigenen Aussagen die lokale Politik nicht besonders gut oder überhaupt nicht, auf der nationalen und internationalen Ebene waren dies immerhin 39\%. Vermutlich schlägt sich in diesen Einstellungen - neben der Überschaubarkeit der meisten Gemeinden - die lange Tradition der kommunalen Selbstverwaltung in Deutschland nieder.

Die subjektive Selbsteinstufung des eigenen Verständnisses darf nicht mit tatsächlich vorhandenem Wissen über kommunalpolitische Fragen verwechselt werden. Selbst wenn man keine allzu hohen Erwartungen an das Informationsniveau der Bevölkerung stellt, vermitteln die hierzu vorliegenden Daten ein recht problematisches Bild der kommunalpolitischen Wirklichkeit. Weder auf der kommunalen noch auf der nationalen Ebene des politischen Systems entspricht die Lehrbuchfigur des wohlinformierten Durchschnittsbürgers der Wirklichkeit. Einer Ende der sechziger Jahre in Frankfurt, Münster und Erlangen durchgeführten Untersuchung zufolge hatte jeder fünfte Befragte überhaupt keine Vorstellungen von den Aufgaben und der Funktionsweise der kommunalen Selbstverwaltung. Jeder zweite konnte nur vage Angaben machen, und lediglich ein knappes Drittel hatte einigermaßen zutreffende Informationen 
über diese Frage. Um die Kenntnis der Aufgaben der Kommunalvertretung war es nicht besser bestellt. Nur ein Drittel der Befragten wies ein einigermaßen zufriedenstellendes Wissen auf und ordnete der Kommunalvertretung Aufgaben wie die Kontrolle der Verwaltung, die Beratung kommunaler Belange, die Betrauung der Verwaltung mit kommunalen Aufgaben oder die Wahl des Bürgermeisters zu (vgl. GABRIEL 1979: 112, 119). Neuere Untersuchungen machen keine Revision dieses Bildes erforderlich, selbst wenn sie noch niedrigere Ansprïche an das Informationsniveau der Bevölkerung stellen. In HILLs (1987: 76 ff.) Untersuchung, in der einige einfache Fragen zu den Personalkenntnissen der Befragten enthalten waren,

"fielen vor allem der relativ geringe Bekanntheitsgrad der Ratsmitglieder sowie häufige Verwechslungen mit der Verwaltungsspitze und die Popularität der Bürgermeister bzw. Hauptverwaltungsbeamten auf. ... Die Zahlen dokumentieren insgesamt eine überaus schlechte Kenntnis der Repräsentanten" (HILL 1987: 76, 79; Hervorh. im Original).

Im Gefolge der Gebietsreform nahm das ohnehin spärliche Wissen noch weiter ab.

Tabelle 2: $\quad$ Verständnis von Fragen der lokalen und nationalen Politik in der Bundesrepublik, 1959 (Angaben: Prozentanteile)

"Wie gut glauben Sie, daß Sie persönlich die wichtigsten nationalen und internationalen Probleme, denen Westdeutschland gegenübersteht, verstehen? Und wie ist es bei den lokalen Angelegenheiten, die nur diese Gemeinde oder diese Gegend hier betreffen? Wie gut verstehen Sie diese? Sehr gut, ziemlich gut, das hängt vom Gegenstand ab, nicht so gut, überhaupt nicht."

Einwohnerzahl der Gemeinde

\begin{tabular}{|r|r|r|r|r|}
\hline bis & 5.000 & 20.000 & 100.000 & $\begin{array}{r}\text { Alle } \\
\text { u.m. }\end{array}$ \\
Befragten \\
\hline
\end{tabular}

Nationale Politik

\begin{tabular}{|l|r|r|r|r|r|}
\hline gut & 42 & 52 & 44 & 56 & 48 \\
kommt darauf an & 7 & 4 & 14 & 8 & 8 \\
nicht gut & 45 & 39 & 36 & 32 & 39 \\
k.A. & 7 & 6 & 5 & 4 & 5 \\
\hline
\end{tabular}

\section{Lokale Politik}

\begin{tabular}{|l|r|r|r|r|r|}
\hline kommt darauf an & 7 & 4 & 14 & 8 & 8 \\
nicht gut & 45 & 39 & 36 & 32 & 39 \\
k.A. & 7 & 6 & 5 & 4 & 5 \\
gut & 68 & 67 & 54 & 54 & 62 \\
kommt darauf an & 5 & 4 & 17 & 9 & 8 \\
nicht gut & 22 & 25 & 24 & 31 & 25 \\
k.A. & 6 & 4 & 5 & 7 & 6 \\
\hline
\end{tabular}

Prozentpunktdifferenz lokal-national

\begin{tabular}{|l|r|r|r|r|r|}
\hline gut & 26 & 15 & 10 & -2 & 14 \\
kommt darauf an & -2 & 0 & 3 & 1 & 0 \\
kicht gut & -23 & -14 & -12 & -1 & -14 \\
& -1 & -2 & 0 & 3 & 1 \\
\hline N & 354 & 180 & 149 & 272 & 955 \\
\hline
\end{tabular}

Quelle: ALMOND/VERBA 1965; Auswertung d. Verfasser; Kategorien v. Verf. zusammengefaßt. 
Es wäre allerdings irreführend, die unzulängliche Informiertheit über politische $\mathrm{Zu}$ sammenhänge als ein exklusives Merkmal der lokalen Politischen Kultur darzustellen. Wie in anderen demokratischen Staaten ist das Wissen der Bevölkenung über Probleme der nationalen Politik in der Bundesrepublik ebenfalls oberflächlich und lückenhaft. Einige interessante Daten hierzu sind in SCHOTTEMEYERs (1986: $118 \mathrm{ff}$.) Untersuchung über die Einstellungen der Bundesbürger zum Deutschen Bundestag zu finden.

Im Hinblick auf das Verständnis nationaler und lokaler Fragen bestehen gewisse Unterschiede zwischen großen und kleinen Kommunen. Die Bewohner kleiner Gemeinden gaben an, lokalpolitische Probleme erheblich besser zu verstehen als Fragen der nationalen Politik. Der Anteil derer, die nach ihren eigenen Angaben kommunale Fragen gut verstanden, lag um 26 Prozent höher als der entsprechende Wert für die nationale und internationale Politik. Darüber hinaus zeigen die in Tabelle 2 enthaltenen Angaben, daß sich die Kluft zwischen dem Verständnis lokaler und überlokaler Angelegenheiten mit zunehmender Gemeindegröße deutlich verkleinerte. Großstädter schätzten die Durchschaubarkeit der Kommunalpolitik und der überlokalen Politik nahezu gleich ein. Sie verfügten über ein wesentlich besseres Verständnis nationaler Fragen als die Bewohner kleiner Gemeinden, gaben aber andererseits an, lokalpolitische Probleme schlechter zu verstehen. Dazwischen liegen die Einstellungen der Mittel- und Kleinstädter. Ausweislich dieser Daten ist die Bürgernähe kein generelles Attribut der Kommunalpolitik, sondern sie zeichnet vor allem kleine Gemeinden aus. Großstädter halten die kommunale Politik nicht für überschaubarer als die "große Politik". Diese Einschätzungen reflektieren das tatsächlich vorhandene Komplexitätsgefälle zwischen der Großstadtpolitik und der Politik in kleinen Gemeinden.

Die hier präsentierten Daten machen deutlich, daß die politische Involvierung keine einheitliche Größe ist: Das Interesse an Fragen der nationalen und lokalen Politik ist in der Bundesrepublik relativ hoch entwickelt. Daneben glaubt die Bevölkerung, politische Vorgänge in der Gemeinde und im Bund einigermaßen gut zu verstehen. Zugleich ist das Wissen über politische Zusammenhänge nur schwach entwickelt. Dieses Einstellungsprofil scheint in der kulturellen Tradition der Bundesrepublik relativ fest verankert zu sein. Bereits ALMOND und VERBA (1965: $53 \mathrm{ff}$.) bescheinigten den Bundesbürgem eine vergleichsweise große Bereitschaft zur Auseinandersetzung mit politischen Vorgängen. Ihrer Meinung nach waren die demokratischen Defizite der Politischen Kultur der Bundesrepublik nicht am politischen Interesse und am politischen Informationsniveau der Bevölkerung festzumachen. Mehrere Untersuchungen der nationalen Politischen Kultur der Bundesrepublik belegen, daß die politische Involvierung der Bundesbürger seit dem Beginn der sechziger Jahre nochmals deutlich zunahm (vgl. BAKER/DALTON/HILDEBRANDT 1981: 38 ff.; GABRIEL 1986: $182 \mathrm{ff}$.). Auch wenn neuere national repräsentative Daten zur Anteilnahme der Bundesbürger an der Kommunalpolitik fehlen, gibt es keinen Grund zu der Vermutung, die kommunalpolitische Involvierung könnte zurïckgegangen sein. Auch aus der Entwicklung der Beteiligung an Kommunalwahlen lassen sich keine Anhaltspunkte für eine derartige Annahme gewinnen. Möglicherweise nahm das Interesse an den Vorgängen in der Gemeinde in Folge der Gebietsreform ab. Damit ergab sich aber eher eine Verschiebung zur Bundespolitik als ein genereller Rückzug der Bevölkerung aus der Politik. 


\section{Übungsaufgabe 2:}

Fassen Sie die wichtigsten Ergebnisse der Forschung zur Gemeindeidentifikation, zum Interesse an der Kommunalpolitik, zum subjektiven Verständnis kommunaler Fragen und zum kommunalpolitischen Wissen thesenartig zusammen!Geben Sie jeweils einen markanten empirischen Beleg für die These an!

\subsection{Kommunalpolitik zwischen Parteipolitik und Verwaltung}

\subsubsection{Die Einschätzung der "Politikfähigkeit" der kommunalen Selbstverwaltung und der Rolle der Parteien in der Kommunalpolitik}

Das Interesse an den Vorgängen in der Kommune und das Verständnis lokaler Probleme muß nicht zwangsläufig politisch ausgerichtet sein. Folgt man den vorherrschenden Einschätzungen, dann verstehen die Bürgerinnen und Bürger die Kommunalpolitik als eine "unpolitische" Angelegenheit (vgl. z.B. LEHMBRUCH 1979: 324 ff.; HOLTMANN 1990: 11 ff.). Sie unterscheiden systematisch zwischen der sachbezogenen Erfüllung kommunaler Verwaltungsaufgaben und der "großen Politik", in der es um die Lösung (partei-)politischer Streitfragen gehe. Diese Vorstellungsmuster sind angeblich fest in der deutschen Selbstverwaltungstradition verwurzelt. Sie äußern sich vor allem in starken Vorbehalten gegenüber der Betätigung der Parteien in der Kommunalpolitik.

Erste Informationen über die Akzeptanz einer parteipolitisch geprägten Kommunalpolitik bzw. über die "Politikfähigkeit" der kommunalen Selbstverwaltung finden sich in der bereits erwähnten Umfrage in Frankfurt, Münster und Erlangen. In ihr votierte eine deutliche Mehrheit der Befragten für ein traditionelles Selbstverwaltungsmodell. Über zwei Drittel vertraten die Auffassung, in der Gemeinde solle nicht nach politischen Gesichtspunkten entschieden werden. Mehr als 60 Prozent stimmten der Aussage zu, die Führung der Gemeinde sollte am besten in die Hände unpolitischer Fachleute gelegt werden (vgl. GABRIEL 1979: 118). Eine starke Minderheit ließ nur ein geringes Verständnis für die demokratischen Attribute der kommunalen Selbstverwaltung erkennen. Zwischen 22 und 47 Prozent stimmten der Auffassung zu, für die wirklichen Ereignisse in der Gemeinde sei es unbedeutend, ob man die Mitglieder der Gemeindevertretung wählen könne oder nicht. Der Aussage, auf die Wahl der Mitglieder der Gemeindevertretung könne man eigentlich verzichten, stimmten zwischen 21 und 38 Prozent der Befragten zu (vgl. GABRIEL 1979: 112 f.). Alle diese Einstellungen lassen deutliche Vorbehalte gegenüber parteienstaatlich-wettbewerbsdemokratischen Prinzipien erkennen.

Allerdings drängt sich die Frage auf, ob die negativen Einstellungen zu parteienstaatlichen Strukturen wirklich nur die lokale Politik betreffen oder sie nicht auch für die nationale Ebene gelten und somit zu den generellen Merkmalen der Politischen Kultur der Bundesrepublik gehören. Unter diesem Gesichtspunkt ist eine Analyse der Einstellungen der Bundesbürger zur Rolle der Parteien in der kommunalen und nationalen Politik aufschlußreich, zumal die Daten einen Vergleich der Situation am Ende der sechziger und der siebziger Jahre zulassen.

Vor zwanzig Jahren betrachtete die Mehrheit der Bundesbürger die politischen Parteien als Störfaktoren oder sachfremde Elemente in der Kommunalpolitik. Nach den von OEL (1972: 87 f., 106) vorgelegten Daten sprachen sich 65 Prozent der Be- 
fragten gegen einen Einfluß der Parteien auf die Kommunalpolitik aus, nur 24 Prozent billigten den Parteien Einfluß auf kommunale Entscheidungen zu. Erheblich positiver war die Bevölkerung bereits damals zur Rolle der Parteien in der Bundespolitik eingestellt: Die Hälfte der Befragten betrachtete die Bundespolitik als ein legitimes Aktionsfeld der Parteien, nur ein Drittel votierte gegen einen Einfluß der Parteien auf die Bonner Politik. Am Ende der sechziger Jahre war der traditionelle Anti-Parteien-Affekt in der Bundespolitik nur noch bei einer Minderheit anzutreffen, dagegen fand die Vorstellung von einer parteifreien Kommunalpolitik immer noch eine breite Unterstützung.

Tabelle 3: Die Einschätzung der Rolle der Parteien in der nationalen Politik, 1979 (Angaben: Prozentanteile).

"Man hört verschiedene Meinungen über die Bedeutung der Rolle politischer Parteien in der Politik. Geben Sie bitte an, wie sehr sie dafür oder dagegen sind, daß politische Parteien in der Politik der Bundesrepublik Deutschland eine sehr wichtige Rolle spielen.

Und in welchem Maße sind Sie dafur oder dagegen, daß politische Parteien hier in der Stadt oder Gemeinde eine sehr wichtige Rolle spielen?"

Einwohnerzahl der Gemeinde

\begin{tabular}{|r|r|r|r|r|}
\hline bis & 5.000 & 20.000 & 100.000 & Alle \\
5.000 & -19.999 & -99.999 & u.m. & Befragten \\
\hline
\end{tabular}

Nationale Politik

\begin{tabular}{|l|r|r|r|r|r|}
\hline dafür & 57 & 58 & 54 & 62 & 58 \\
unentschieden & 23 & 18 & 17 & 18 & 19 \\
dagegen & 5 & 8 & 4 & 4 & 5 \\
k.A. & 15 & 16 & 25 & 16 & 18 \\
\hline
\end{tabular}

Lokale Politik

\begin{tabular}{|l|r|r|r|r|r|}
\hline dafür & 43 & 44 & 48 & 54 & 48 \\
unentschieden & 24 & 22 & 17 & 19 & 20 \\
dagegen & 20 & 19 & 13 & 9 & 15 \\
k.A. & 13 & 15 & 22 & 18 & 17 \\
\hline
\end{tabular}

Prozentpunktdifferenz lokal-national

\begin{tabular}{|l|r|r|r|r|r|}
\hline dafür & -14 & -14 & -6 & -8 & -10 \\
unentschieden & 1 & 4 & 0 & 1 & 1 \\
dagegen & 15 & 11 & 9 & 5 & 10 \\
k.A. & 2 & -1 & -3 & 2 & -1 \\
\hline \hline $\mathrm{N}$ & 197 & 245 & 245 & 316 & 1003 \\
\hline
\end{tabular}

Quelle: Eurobarometer 11; Auswertung d. Verf.

Bis zum Ende der siebziger Jahre festigten die Parteien ihre Position auf allen Ebenen des politischen Systems der Bundesrepublik. Dies ergibt sich nicht allein aus der Mitgliederentwicklung und aus dem Wählerverhalten (zur nationalen Politik vgl. z.B. die Angaben bei RUDZIO 1991: 158 ff.; für die lokale Ebene GABRIEL 1975: 3 ff.). Auch 
die Einstellung der Bundesbürger zu den politischen Parteien entwickelte sich positiv. Nach den in Tabelle 3 enthaltenen Daten wuchs die Akzeptanz parteienstaatlicher Strukturen auf der nationalen, vor allem aber auf der lokalen Ebene des politischen Systems. Mehr als die Hälfte der Befragten, nämlich 58 Prozent, wies den Parteien eine wichtige Rolle in der nationalen Politik zu. Das waren acht Prozent mehr als in OELS Untersuchung. Zwar beurteilten die Bundesbürger die Aktivitäten der Parteien auf der lokalen Ebene etwas kritischer, doch waren positive Einstellungen zu den Parteien in der Kommunalpolitik wesentlich weiter verbreitet als negative Bewertungen (48 bzw. 15\%; vgl. ergänzend auch die Daten bei BIEGE et al. 1978: 158 ff, bes. Tabelle 34; HILL 1987: 235, Tabelle 129). Im Vergleich mit OELs Untersuchung läßt sich eine Verdoppelung des Anteils positiver Einstellungen feststellen. Noch deutlicher zeigt sich das Ausmaß des Wandels freilich an der Entwicklung der parteienkritischen Einstellungen. Sie gingen von $65 \%$ auf $15 \%$ zurïck. Auch wenn die Formulierung der Fragen und der Antwortvorgaben in den beiden Untersuchungen nicht vollständig identisch war und hieraus gewisse Probleme hinsichtlich der Vergleichbarkeit der Daten resultieren, ist der Entwicklungstrend eindeutig: Die Akzeptanz der Parteien als Träger in der Kommunalpolitik nahm zu, der Anti-Parteien-Affekt wurde abgebaut.

Als Folge dieses Einstellungswandels glich sich die lokale der nationalen Politischen Kultur an. Während die Bevölkerung die Rolle der Parteien in der Bundes- und in der Kommunalpolitik zunächst noch sehr unterschiedlich einschätzte, differierten diese Einstellungen am Beginn der achtziger Jahre nur noch graduell. Die meisten Bundesbürger nehmen die Bundes- und die Lokalparteien als eine Einheit wahr. Für diese Annahme spricht auch die starke positive Beziehung zwischen der Einstellung zur kommunal- und bundespolitischen Rolle der Parteien. Nur eine kleine Minderheit unter den Bundesbürgern spricht sich gegen eine Mitwirkung der Parteien an der Bundespolitik und der Kommunalpolitik aus, allerdings gibt es bezüglich der lokalpolitischen Rolle der Parteien eine vergleichsweise große Zahl Indifferenter und Meinungsloser.

Die Größe des Wohnortes der Befragten wirkt sich auf seine Einstellung zur Rolle der Parteien in der Bundespolitik und Kommunalpolitik erstaunlich wenig aus. Die Bewertung der bundespolitischen Aktivitäten der Parteien variiert überhaupt nicht systematisch mit der Gemeindegröße. Den Parteien wird zwar mit zunehmender Gemeindegröße ein größerer Einfluß auf die Kommunalpolitik zugebilligt, doch haben wir es eher mit graduellen als mit grundsätzlichen Einstellungsunterschieden zu tun. Im Einklang mit diesen Befunden steht der Tatbestand, daß die politischen Parteien heute selbst in kleinen Gemeinden weitgehend die kommunalen Entscheidungsgremien kontrollieren.

\subsubsection{Die Einstellungen zur Kommunalverwaltung}

Ein zweites Merkmal der traditionellen Konzeption kommunaler Selbstverwaltung sehen Beobachter in der hohen Wertschätzung einer unparteiischen, funktionsfähigen und kompetenten Verwaltung. In der politischen Vorstellungswelt vieler Bürgerinnen und Bürger bildet sie den Gegenpol zu den politischen Parteien. Allerdings betrachteten ALMOND und VERBA (1965: 45 ff., 126 ff., 140 ff., $171 \mathrm{ff}$., $312 \mathrm{f}$.) dieses Einstellungsmuster als ein generelles Merkmal der Politischen Kultur Deutschlands. Sie stützten deren Charakterisierung als Untertanenkultur hauptsächlich auf das festge- 
stellte Ungleichgewicht zwischen den Input- und den Output-Orientierungen. Nach ihren Aussagen standen die meisten Bundesbürger den Institutionen und Verfahren partizipativer Politik negativ oder gleichgültig gegenüber, zugleich ließen sie eine hohe Wertschätzung der Exekutivinstitutionen erkennen.

Die ersten mir zugänglichen national repräsentativen Daten zur Bewertung der lokalen Verwaltung durch die Bundesbürger entstammen der Civic-Culture Studie. Für das Jahr 1984 liegen ebenfalls Daten zur Zufriedenheit mit der Kommunalverwaltung vor. Aus der Mehrdeutigkeit des Begriffs "Kommunalverwaltung" ergeben sich gewisse Unsicherheiten bei der Interpretation dieser Daten. Im verfassungsrechtichen Sinne gehören alle Entscheidungsgremien der Gemeinde, d.h. die Gemeindevertretung, der Bürgermeister und die Gemeindeverwaltung im engeren Sinne zur Kommunalverwaltung. $\mathrm{Ob}$ auch die Bevölkerung den Begriff in diesem weiten Sinne interpretiert oder ob sie ihn auf die kommunale Exekutive im engeren Sinne begrenzt, kann in unserem Zusammenhang nicht geklärt werden. Deshalb läßt sich nicht genau angeben, auf welche Dimension der lokalen Politischen Kultur sich die Fragen nach der Kommunalverwaltung beziehen. Klar ist allerdings, daß die in der Civic-CultureStudie enthaltenen Indikatoren auf die Leistungen der Exekutive für die Bürger, also auf die regulativen Aspekte der lokalen Politik zielen. Da in der 1984 benutzten Frage der Leistungsbezug fehlte und der Begriff "Zufriedenheit" sehr weit gefaßt ist, muß offen bleiben, unter welchem Gesichtspunkt die Befragten die Kommunalverwaltung bewerteten. Ihre Aussagen können eine positive Einstellung zu den Leistungen der kommunalen Institutionen widerspiegeln und sich damit auf die regulativen Aspekte der lokalen Politik beziehen, aber auch ein generalisiertes Vertrauen zu ihnen erfassen, d.h. die generelle Einstellung zur Institution kommunale Selbstverwaltung oder die Regimeunterstützung im Sinne EASTONs.

Im Jahre 1959 war die lokale und die nationale Exekutive von den Bundesbürgern unter zwei Gesichtspunkten zu bewerten. Zunächst war zu beurteilen, wie groß der Einfluß ihrer Aktivitäten auf die Lebensbedingungen der Bevölkerung war. Darüber hinaus waren die positiven oder negativen Effekte ihrer Tätigkeit einzuschätzen. Die Bedeutsamkeit der Tätigkeit der Bundes- und Kommunalbehörden stuften die Befragten ziemlich ähnlich ein: 38\% schrieben der Bundespolitik eine große Bedeutung für ihr eigenes Leben zu, 33\% hielten die Kommunalpolitik für bedeutsam. Nur eine kleine Minderheit von 17 bzw. 18\% vertrat die Auffassung, bundes- und kommunalpolitische Entscheidungen beeinflußten ihre persönlichen Lebensbedingungen nicht. Diese Verteilung fällt in Gemeinden unterschiedlicher Größe tendenziell ähnlich aus, zumindest läßt sich keine klare systematische Beziehung zwischen der Gemeindegröße und der Bedeutsamkeit der Politik bzw. Verwaltung ausmachen (vgl. Tabelle 4). Für die Mehrheit der Bundesbürger stellen die Kommunal- und die Bundespolitik feste Größen in ihrem Leben dar.

In der Sicht der meisten Bürgerinnen und Bürger waren die Institutionen des Staates und der Gemeinde 1959 nicht nur bedeutsam für das eigene Leben, darüber hinaus schrieb man ihnen auch eine positive Rolle zu. $60 \%$ der Befragten gaben an, die Aktivitäten der lokalen politischen Führung bewirkten eine Verbesserung der eigenen Lebensbedingungen, den nationalen Regierungsinstitutionen bescheinigten fast ebenso viele, nämlich 56\%, diese Eigenschaft. Nur drei bzw. ein Prozent sahen in den Aktivitäten nationaler und lokaler Instanzen eine Beeinträchtigung ihrer Lebensverhältnisse (vgl. Tabelle 5). In dieser außerordentlich positiven Einstellung zu den regulativen Aspekten der Politik sahen ALMOND und VERBA (1985: 182 f.) ein typi- 
sches, durch die neuere geschichtliche Entwicklung bedingtes Merkmal der Politischen Kultur Deutschlands. Sie machten die frühzeitige Institutionalisierung rechts-, sozial- und leistungsstaatlicher Prinzipien dafür verantwortlich, daß die Deutschen die Politik vornehmlich über die staatlichen Outputs und deren Träger wahrnähmen. Für das Verhältnis zur Kommunalverwaltung ist diese Interpretation ebenfalls plausibel; denn vor der endgültigen Demokratisierung des nationalen politischen Systems waren auf der kommunalen Ebene demokratisch-partizipative Einrichtungen zwar formal vorhanden, praktisch aber nur bedingt funktionsfähig. Dagegen erfüllten die Kommunen lange vor der Gründung der Bundesrepublik umfassende Leistungen auf dem Gebiet der Daseinsvorsorge.

Tabelle 4: $\quad$ Existenz von Auswirkungen der Tätigkeit der nationalen und lokalen politischen Führung auf die Lebensbedingungen der Bevölkerung, 1959 (Angaben: Prozentanteile).

"Wenn wir nun einmal an die Bundesregierung in Bonn denken, welche Auswirkungen hat die Tätigkeit der Bundesregierung, das Erlassen von Gesetzen usw. auf Ihr tägliches Leben? Hat sie wesentliche Auswirkungen, einige oder gar keine Auswirkungen?

Nehmen wir nun einmal die Gemeindeverwaltung. Was glauben Sie, welche Auswirkungen haben deren Maßnahmen auf Ihr tägliches Leben? Haben sie wesentliche Auswirkungen, einige oder gar keine Auswirkungen?"

Einwohnerzahl der Gemeinde

\begin{tabular}{|c|r|r|r|r|}
\hline bis & 5.000 & 20.000 & 100.000 & $\begin{array}{r}\text { Alle } \\
\text { u.m. }\end{array}$ \\
\hline Befragten \\
\hline
\end{tabular}

Nationale Politik

\begin{tabular}{|l|r|r|r|r|r|}
\hline wesentl. Auswirkungen & 36 & 38 & 35 & 43 & 38 \\
einige Auswirkungen & 33 & 32 & 30 & 34 & 33 \\
keine Auswirkungen & 15 & 19 & 22 & 14 & 17 \\
k.A. & 16 & 11 & 14 & 9 & 13 \\
\hline
\end{tabular}

Lokale Politik

\begin{tabular}{|l|r|r|r|r|r|}
\hline wesentl. Auswirkungen & 34 & 29 & 28 & 37 & 33 \\
einige Auswirkungen & 39 & 44 & 38 & 42 & 41 \\
keine Auswirkungen & 19 & 18 & 20 & 15 & 18 \\
k.A. & 8 & 8 & 13 & 6 & 9 \\
\hline
\end{tabular}

Prozentpunktdifferenz lokal-national

\begin{tabular}{|l|r|r|r|r|r|}
\hline wesentl. Auswirkungen & -2 & -9 & -7 & -6 & -5 \\
einige Auswirkungen & 6 & 12 & 8 & 8 & 8 \\
keine Auswirkungen & 4 & -1 & -2 & 1 & 1 \\
k.A. & -8 & -3 & -1 & -3 & -4 \\
\hline \hline N & 354 & 180 & 149 & 272 & 955 \\
\hline
\end{tabular}

Quelle: ALMOND/VERBA 1965; Auswertung d. Verf. 
Tabelle 5: $\quad$ Art der Auswirkungen der Tätigkeit der nationalen und lokalen politischen Führung auf die Lebensbedingungen der Bevölkerung, 1959 (Angaben: Prozentanteile).

"Im großen und ganzen gesehen: Führt die Tätigkeit der Bundesregierung dazu, die Zustände in Deutschland zu verbessem oder ginge es uns ohne sie wesentlich besser?

Im großen und ganzen gesehen: Führt die Tätigkeit der Gemeindeverwaltung dazu, die $\mathrm{Zu}$ stände in dieser Gegend zu verbessern oder ginge es besser ohne sie?"

Einwohnerzahl der Gemeinde

\begin{tabular}{|r|r|r|r|r|}
\hline bis & 5.000 & 20.000 & 100.000 & $\begin{array}{r}\text { Alle } \\
\text { u.000 }\end{array}$ \\
& -19.999 & -99.999 & u.m. & Befragten \\
\hline
\end{tabular}

Nationale Politik

\begin{tabular}{|l|r|r|r|r|r|}
\hline Verbesserungen & 59 & 72 & 40 & 50 & 56 \\
teils/teils, neutral & 31 & 20 & 36 & 35 & 31 \\
Verschlechterungen & 1 & 2 & 5 & 4 & 3 \\
k.A. & 9 & 7 & 20 & 11 & 11 \\
\hline
\end{tabular}

\section{Lokale Politik}

\begin{tabular}{|l|r|r|r|r|r|}
\hline Verbesserungen & 65 & 76 & 48 & 50 & 60 \\
teils/teils, neutral & 27 & 18 & 36 & 40 & 30 \\
Verschlechterungen & 1 & 1 & 1 & 1 & 1 \\
k.A. & 7 & 6 & 16 & 9 & 9 \\
\hline
\end{tabular}

Prozentpunktdifferenz lokal-national

\begin{tabular}{|l|r|r|r|r|r|}
\hline Verbesserungen & 6 & 4 & 8 & 0 & 4 \\
teils/teils, neutral & -4 & -2 & -0 & 5 & -1 \\
Verschlechterungen & 0 & -1 & -4 & -3 & -2 \\
k.A. & -2 & -1 & -4 & -2 & -2 \\
\hline N & 354 & 180 & 149 & 272 & 955 \\
\hline
\end{tabular}

Quelle: ALMOND/VERBA 1965; Auswertung d. Verf.

Die Bewohner von Gemeinden aller Größenklassen schätzten die Auswirkungen der Regierungstätigkeit auf das eigene Leben mehrheitlich positiv ein. Diese Einstellung stellte somit ein durchgängiges Merkmal der lokalen und der nationalen Politischen Kultur der Bundesrepublik dar. Allerdings schnitten die lokalen politischen Institutionen im Urteil der Klein- und Mittelstädter etwas besser ab als Einrichtungen der nationalen Politik, in Großstädten dagegen wurden beide etwa gleich positiv beurteilt (vgl. Tabelle 5).

Obgleich 1984 ein anderes Erhebungsinstrument eingesetzt wurde als in der Civic-Culture-Studie, beziehen sich die in den beiden Umfragen benutzten Indikatoren auf thematisch ähnliche Sachverhalte. Ihre Ergebnisse dürften deshalb mit gewissen Einschränkungen vergleichbar sein. Auch im Jahre 1984 waren die meisten Bundesbürger positiv zur Kommunalverwaltung eingestellt. $68 \%$ der Befragten bezeichneten die Art und Weise, wie die Kommunalverwaltung ihre Aufgaben erfülle, als ziemlich gut, $28 \%$ bewerteten die Arbeit der Kommunalverwaltung als nicht so gut. Instruktiv ist in diesem Zusammenhang ein Vergleich mit den Einstellungen zum nationalen 
politischen System. Die Frage nach der Zufriedenheit mit dem Funktionieren der Demokratie in der Bundesrepublik beantworteten in der gleichen Erhebung $71 \%$ der Befragten positiv (vgl. Tabelle 6). Die Zufriedenheit mit der Kommunalverwaltung unterscheidet sich also nicht wesentlich von der Zufriedenheit mit der Demokratie in der Bundesrepublik. Zudem hängen beide Einstellungen miteinander zusammen, auch wenn die Beziehung zwischen ihnen nicht besonders stark ausgeprägt ist.

Tabelle 6: Einstellung zur Kommunalverwaltung und zur Demokratie in der Bundesrepublik, 1984 (Angaben: Prozentanteile).

"Sind Sie mit der Art und Weise, wie die Demokratie in der Bundesrepublik funktioniert, alles in allem gesehen sehr zufrieden, ziemlich zufrieden, ziemlich unzufrieden oder völlig unzufrieden?"

"Hier auf dieser Liste stehen einige Dinge, die Ihr tägliches Leben beeinflussen. Können Sie mir bitte für jedes sagen, ob es Ihrer Meinung nach ziemlich gut oder nicht so gut funktionien?

-...;

.... die ... An und Weise, wie Ihre Kommunalverwaltung ihre Aufgaben erfüllt; $-. . . "$

Einwohnerzahl der Gemeinde

\begin{tabular}{|c|r|r|r|r|}
\hline bis & 5.000 & 20.000 & 100.000 & Alle \\
5.000 & -19.999 & -99.999 & u.m. & Befragten \\
\hline
\end{tabular}

Demokratie in BRD

\begin{tabular}{|l|r|r|r|r|r|}
\hline zufrieden & 75 & 69 & 76 & 66 & 71 \\
nicht zufrieden & 16 & 23 & 18 & 26 & 22 \\
k.A. & 9 & 7 & 6 & 7 & 7 \\
\hline
\end{tabular}

Kommunalverwaltung

\begin{tabular}{|l|r|r|r|r|r|}
\hline zufrieden & 74 & 75 & 66 & 62 & 68 \\
nicht zufrieden & 22 & 22 & 33 & 33 & 28 \\
k.A. & 5 & 4 & 1 & 4 & 3 \\
\hline
\end{tabular}

Prozentpunktdifferenz Kommune-Staat

\begin{tabular}{|l|r|r|r|r|r|}
\hline zufrieden & -1 & 6 & -10 & -4 & -3 \\
nicht zufrieden & 6 & -1 & 15 & 7 & 6 \\
k.A & -4 & -3 & -5 & 3 & -4 \\
\hline
\end{tabular}

Quelle: Eurobarometer 21; Auswertung d. Verf.; N=955.

Während die Einstellung zur Demokratie in der Bundesrepublik in Gemeinden unterschiedlicher Größe nur geringfügig vom nationalen Durchschnittswert abweicht, ist die Zufriedenheit mit der Kommunalverwaltung in Kleinstgemeinden und Kleinstädten etwas stärker ausgeprägt als in Mittel- und Großstädten (vgl. Tabelle 6). Es wäre genauer zu prüfen, ob dieser Befund den besonderen Problemdruck widerspiegelt, dem die Großstadtpolitik im Vergleich mit der Politik in kleineren Gemeinden ausgesetzt ist. Die hierfür benötigten Daten stehen aber nicht zur Verfügung.

Im Hinblick auf die deutsche Selbstverwaltungstradition, in der administrativen Orientierungen immer ein Übergewicht gegenüber parteienstaatlichen Einstellungen zukam, ist ein Vergleich zwischen der Zufriedenheit mit der Kommunalverwaltung 
und der Bewertung der Rolle der Parteien in der Kommunalpolitik aufschlußreich. Bei der Interpretation dieser Daten muß man allerdings berücksichtigen, daß die Einstellungen zu diesen beiden wichtigen Trägern des kommunalpolitischen Willensbildungs- und Entscheidungsprozesses zu unterschiedlichen Zeitpunkten und auf verschiedenen Bewertungsdimensionen erhoben wurden. Zumindest in einer Hinsicht jedoch lassen sich die Angaben miteinander vergleichen. Die Zufriedenheit mit einer Institution oder Organisation und die Bereitschaft, ihr eine wichtige Rolle in der Politik zuzuweisen, reflektiert eine positive Einstellung zu den betreffenden Objekten. Während mehr als zwei Drittel der Bundesbürger positiv zur Kommunalverwaltung eingestellt waren, äußerte nur knapp die Hälfte der Befragten positive Einstellungen zu den Lokalparteien. Diese Verteilung kann man wohl als Hinweis auf die fortdauernde Wirksamkeit traditioneller kommunalpolitischer Doktrinen werten. Administrative Einrichtungen scheinen in der Bevölkerung nach wie vor eine höhere Wertschätzung zu genießen als die Parteien. Erwartungsgemäß dominieren in kleinen Gemeinden administrative gegenüber parteienstaatlichen Orientierungen erheblich stärker als in Großstädten (vgl. im einzelnen die Angaben in den Tabellen 3 und 6).

Bedauerlicherweise erfassen die bislang präsentierten Daten die Beziehungen der Bürgerinnen und Bürger zu ihrer Gemeinde ziemlich unspezifisch. Sie liefern keine differenzierten Informationen über die für die Bewertung der kommunalen Institutionen, der Kommunalverwaltung oder der Parteien maßgeblichen Motive. Da national repräsentative neuere Daten zu diesem Problem nicht vorliegen, ist es erforderlich, auf Befunde aus älteren Untersuchungen und auf Einzelfallstudien zurückzugreifen. Dies ist natürlich nicht unproblematisch; denn es kann nicht unterstellt werden, da $B$ die am Ende der sechziger Jahre typischen Einstellungsmuster auch heute noch Bestand haben und daß die in einzelnen Städten ermittelten Einstellungen für die gesamte Bundesrepublik typisch sind.

Die bereits erwähnte Untersuchung OELs enthielt einige Angaben über das der Kommunalpolitik und der Bundespolitik zugeschriebene Funktionsprofil. OEL unterschied zwischen fünf verschiedenen Sichtweisen der lokalen und nationalen Politik: Nach seiner Typologie konnte bei der Wahrnehmung der Politik der Aspekt der anonymen Herrschaft, der Versorgung mit Gütern und Dienstleistungen, des Entscheidungshandelns, des Interessenausgleichs oder der Autorität und des Schutzes im Vordergrund stehen. Leider zwang das Erhebungsverfahren die Befragten dazu, sich auf eine dieser fünf Alternativen festzulegen. Diese Einschränkung dürfte den tatsächlichen politischen Orientierungen kaum gerecht werden; denn es ist ohne weiteres denkbar, daß die Bevölkerung der Politik zugleich die Funktionen des Interessenausgleichs, des Entscheidungshandelns usw. zuweist. Möglicherweise ergibt sich aus dem Meßverfahren eine verzerrte Verteilung der Antworten.

Die für Deutschland angeblich typische obrigkeits- und leistungsstaatliche Politiktradition legt die Erwartung nahe, daß die Einstellung der Bevölkerung zur Kommunal- und zur Staatspolitik primär durch die Autoritäts- und Schutzfunktion und den Versorgungsaspekt geprägt ist. Nach den in Tabelle 7 enthaltenen Daten traf dies allerdings nur bedingt zu. Erwartungsgemä $B$ entfielen die weitaus meisten Nennungen auf die Autoritäts- und Schutzfunktion der Kommunalpolitik. Mit großem Abstand folgten die Funktionen des Entscheidungshandelns und des Interessenausgleichs. Dagegen spielte die Versorgungskomponente überraschenderweise nur für wenige $\mathrm{Be}$ fragte eine Rolle. Lediglich den Aspekt der anonymen Herrschaft brachten noch weniger Bundesbürger mit der Kommunalpolitik in Verbindung. Wie OEL (1972: 115 f.) 
zutreffend feststellte, schrieb die Bevölkerung der Bundespolitik ein ziemlich ähnliches Funktionenprofil zu: Auch hier stand die Autoritäts- und Schutzfunktion im Vordergrund, gefolgt von den Aufgaben der Entscheidung und des Interessenausgleichs. Einmal mehr zeigt sich, daß sich die Bewertung lokaler und nationaler Institutionen eher graduell als grundsätzlich voneinander unterscheidet. Es ist allerdings nicht möglich, aus diesen Befunden eine Vorherrschaft traditioneller Output-Orientierungen herauszulesen; denn die Möglichkeit, die Politik über die Attribute Partizipation und politischer Wettbewerb zu definieren, bestand für die Befragten nicht.

Tabelle 7: $\quad$ Bewertung der Funktion der lokalen und der nationalen Politik in der Bundesrepublik (Angaben: Prozentanteile).

$\begin{array}{lccc}\text { Anonyme Herrschaft } & \text { Bund } & \text { Gemeinde } & \begin{array}{c}\text { Prozentpunkt- } \\ \text { differenz }\end{array} \\ \text { Versorgung } & 8 & 4 & -4 \\ \text { Entscheidung } & 7 & 7 & 0 \\ \text { Interessenausgleich } & 13 & 19 & 6 \\ \text { Autorität und Schutz } & 12 & 15 & 3 \\ \text { k.A. } & 40 & 30 & -10 \\ & 22 & 25 & 3\end{array}$

Berechnungen durch den Verfasser auf der Basis der Angaben bei OEL 1972: $114 \mathrm{ff}$.; $\mathrm{N}=1801$. Angaben zum Erhebungsinstrument:

Folgende Fragen wurden gestellt:

"Über das, was in der großen Politik geschieht oder geschehen sollte, gibt es verschiedene Meinungen. Welche kommt Ihrer am nächsten?

In den örtlichen Angelegenheiten ist es ja manchmal anders als in der großen Politik. Uber das, was im Rathaus (im Gemeindeamt) vor sich geht, hätte ich auch gern lhre Meinung. Welche kommt Ihrer eigenen Ansicht am nächsten?"

Folgende Antwortmöglichkeiten wurden gegeben:

"Die da oben machen mit uns doch, was sie wollen. Sie lassen sich nicht in die Karten gucken (anonyme Herrschaft).

Die Politiker müssen dafür sorgen, daß man kriegen kann, was man braucht. Dabei ist es nicht so wichtig, wer die Leute sind, die das machen (Versorgung).

Die Männer und Frauen, denen die Bürger bei der Wahl das Vertrauen ausgesprochen haben, sollten für die nächsten Jahre entscheiden, was geschieht (Entscheidung).

In der Politik muß man vor allem darauf achten, daß alle Gruppen zum Zuge kommen und keine auf Kosten der anderen zu mächtig wird (Interessenausgleich).

Die Verantwortlichen sollten dafür sorgen, daß sich jeder sicher fühlen kann und daß jedem sein Recht gewährleistet wird (Autorität und Schutz)."

Weitere interessante Informationen über die Beziehung der Bürgerinnen und Bürger zur Gemeinde liefern kommunalwissenschaftliche Fallstudien. In einer von ARZBERGER (1980: 61 ff., $110 \mathrm{ff}$.) durchgeführten Untersuchung in vier Kleinstädten und in einem Frankfurter Stadtteil gab die Mehrheit der Bevölkerung an, die Vertreter der Stadtverwaltung bemühten sich bei ihren Entscheidungen darum, die Wünsche der Bürger zu erfahren und zu berücksichtigen. Außer in Frankfurt billigte man ihnen dariber hinaus mehrheitlich zu, sich am Wohl der Bürger und nicht am Eigeninteresse zu orientieren. Andere Eigenschaften der Kommunalverwaltung, wie ihre Unbestechlichkeit, Unabhängigkeit und Leistungsorientierung, wurden in allen fünf Städten kontrovers beurteilt. Negative Urteile über die "Klüngelwirtschaft" zwischen Verwaltung, Wirtschaft und Parteien traten fast ebenso häufig auf wie positive Aussagen (vgl. auch JARREN et al. 1989: 247). Ähnlich ambivalente Einschätzungen von 
Parteien, Politikern und Verwaltung ermittelten auch BUSE/NELLES/OPPERMANN (1978: $228 \mathrm{ff}$.) in ihrer Andernach-Studie. Allerdings bestanden gegenüber den Politikern und den Parteien stärkere Vorbehalte als gegenüber der Verwaltung. In den von HILL (1987: 79 ff.) untersuchten Gemeinden gaben mehr als die Hälfte der Befragten an, sie könnten im Bedarfsfalle Mitglieder der Kommunalvertretung erreichen und ihnen ihre Anliegen vortragen, aber nicht einmal ein Drittel sah die persönlichen Belange durch die gewählten Ratsmitglieder ausreichend vertreten. Positive Aussagen konzentrierten sich auf die Vertreter des eigenen Ortsteils. Die Effekte der kommunalen Gebietsreform wurden in den einzelnen Kommunen recht unterschiedlich bewertet, generell kann man aber nicht von einer verschlechterten Interessenrepräsentation sprechen. Schon in mehreren älteren Städtestudien war die Arbeit der Kommunalvertretung im allgemeinen relativ gut bewertet worden, was aber eine kritische Stellungnahme zu einzelnen Aspekten nicht ausschloß (vgl. die Zusammenfassung bei GABRIEL 1979: 117 ff.).

Die zuvor charakterisierten Einstellungen werden in der Literatur als politisches Vertrauen beschrieben (vgl. ausführlicher: GABRIEL 1986: 258 ff.). Nach den bisher vorliegenden Forschungsergebnissen bestehen zwischen den Bürgern und der lokalen Exekutive bzw. der kommunalen Vertretungskörperschaft ambivalente Beziehungen. Ein Teil der Bürger vertraut den kommunalen Entscheidungsträgern, ein anderer Teil mißtraut ihnen. Einige Aspekte der Verwaltungsarbeit werden positiver bewertet als andere. Politisches Vertrauen kann durch konkrete sachliche Leistungen oder durch die allgemeine Art der Amtsführung gebildet werden. ARZBERGER (1980: 113) führt die ambivalente Einstellung zur kommunalen Exekutive nicht auf ein diffuses Gefühl oder allgemeinen Verdruß zurück, sondem auf die Bewertung der städtischen Leistungen, Maßnahmen oder Einrichtungen:

"Ist man zufrieden und hat demzufolge nur ein geringes $\mathrm{Ma} B$ an Forderungen, urteilt man auch uber die Stadtverwaltung einigermaßen positiv; ist man eher unzufrieden und hat man klar umrissene, nicht erfüllte Forderungen, fällt das Urteil eher negativ aus".

$\mathrm{Ob}$ diese leistungsbezogene Interpretation wirklich tragfähig ist, läßt sich ohne weitere detaillierte Analysen der Bestimmungsfaktoren des politischen Vertrauens nicht feststellen.

In der Tradition der Community-Power-Studie (vgl. ARZBERgER 1980: $24 \mathrm{ff}$.)stehen die Fragen nach dem Einfluß einzelner Personen, Organisationen und Institutionen auf die Gestaltung der Kommunalpolitik. In unserem Zusammenhang ist dabei vor allem die Frage interessant, ob die Bevölkerung einzelnen lokalen Organisationen oder Institutionen zu viel oder zu wenig Macht zuschreibt. In ARZBERGERs (1980: 59 ff.) Studie waren alle wichtigen Träger des lokalpolitischen Willensbildungs- und Entscheidungsprozesses unter diesem Gesichtspunkt zu bewerten. Die Parteigremien gehörten in allen fünf Städten zu denjenigen Einrichtungen, deren Macht man als zu groß einschätzte. In zwei Städten entfielen auf die Parteigremien sogar die meisten Nennungen. Obgleich die Einstellungen zur Stadtverwaltung uneinheitlicher ausfielen, wurde auch ihr (bzw. in Frankfurt ihren Einheiten) überwiegend zuviel Macht zugesprochen. Kritisiert wurde dagegen in vier der fünf Städte der zu geringe Einfluß des Stadtparlaments bzw. seiner Fraktionen und Ausschüsse.

$\mathrm{Da}$ diese Bewertungen sicherlich von einem bestimmten Grundverständnis kommunaler Selbstverwaltung getragen sind, kann von einer ungebrochenen Wirksamkeit eines administrativ geprägten Selbstverwaltungsverständnisses wohl kaum die Rede 
sein. Die Kritik der Bürgerschaft richtet sich nicht nur auf die Macht der Parteien als Repräsentanten eines neuen politischen Stils, sondern auch auf die Stellung der Verwaltung als Säule des traditionellen Selbstverwaltungsverständnisses. In der Forderung nach mehr Einfluß für die gewählte Stadtvertretung kommt vermutlich eine Aufwertung der demokratisch-partizipativen Komponente der Kommunalpolitik zum Ausdruck. Kommunalpolitik stellt sich für die Bürgerinnen und Bürger nicht nur als Parteipolitik, aber auch nicht ausschließlich als Bewältigung sachrationaler Verwaltungsaufgaben dar. Sie wird als Bestandteil einer gewaltenteiligen, parteienstaatlichen, repräsentativ verfaßten Demokratie gesehen, zu der Parteien, Parlament und Verwaltung gehören.

Übungsaufgabe 3:

Skizzieren Sie die Entwicklung der Einstellungen zur Rolle der Parteien in der Kommunalpolitik und zur Kommunalverwaltung!

\subsubsection{Politische Institutionen, Parteipolitik und Verwaltung aus der Sicht des lokalpolitischen Führungspersonals}

Während über die Einstellungen der Bevölkerung zur Funktionsweise und zu den Institutionen der kommunalen Selbstverwaltung nur verhältnismäßig globale Informationen vorliegen, gibt es mehrere vergleichende Untersuchungen der politischen Einstellungen kommunaler Führungsgruppen, die differenzierte Daten über die Wahrnehmung und Bewertung der Eigenschaften der kommunalen Selbstverwaltung enthalten. Im Vergleich mit den Bevölkerungsumfragen haben sie darüber hinaus den Vorzug, die Perspektive derjenigen Personen wiederzugeben, die maßgeblich den Ablauf und das Ergebnis lokalpolitischer Entscheidungen beeinflussen.

Diese ausschließlich in den achtziger Jahren veröffentlichten Untersuchungen belegen zwar einerseits die ungebrochene Wirksamkeit der traditionellen deutschen Selbstverwaltungskonzeption, sie zeigen aber auch Abweichungen von diesem $\mathrm{Mu}$ ster. Demnach vertreten die Inhaber kommunaler Führungspositionen keine einheitliche Auffassung von der Rolle der kommunalen Selbstverwaltung. Zwar verstehen sie die Kommunalpolitik mehrheitlich als sachbezogene, konflikt-, ideologie- und parteiferne Regelung von Verwaltungsaufgaben. Eine starke Minderheit räumt jedoch die Existenz parteipolitischer Kontroversen ein und bewertet diese nicht unbedingt als dysfunktional. In einer Befragung von Angehörigen der Minderheitsfraktionen in nordrhein-westfälischen Großstädten stimmten $70 \%$ der Respondenten der Auffassung zu, im Stadtrat müsse die Sacharbeit den Vorrang vor der Parteipolitik haben, nur $18 \%$ lehnten diese Auffassung ausdrücklich ab. Auf der anderen Seite war die "Oppositionsfähigkeit" der Kommunalpolitik umstritten. Im Einklang mit dem traditionellen, harmonieorientierten Modell der Kommunalpolitik sprachen sich 39\% der Befragten gegen eine harte Oppositionspolitik und für Einigkeit zwischen allen Kräften in der Gemeinde aus. Die gegenteilige Auffassung fand aber mit einem Anteil von 45\% der Nennungen eine noch etwas breitere Unterstützung (vgl. GABRIEL 1984: 7; ausführlicher zur CDU-Teilerhebung: GABRIEL/HAUNGS/ZENDER 1984: $119 \mathrm{ff}$. , bes. Tabelle 4-1; vgl. auch KÖSER/CASPERS-MERK 1989; JARREN et al. 1989: 246 ff.; SIMON 1988: bes. 95 ff.; WeHLING/SIEWERT 1987: 79 ff.). Obgleich die Mehrheit der jungen, formal gut gebildeten Ratsmitglieder für das traditionelle, verwaltungsorientierte Selbstverwaltungsmodell votierte, signalisiert die überdurchschnittliche Präfe- 
renz dieser Gruppe für eine stärker parteienstaatliche Kommunalpolitik einen Wandel der Selbstverwaltungsdoktrin (vgl. GABRIEL 1984: 9 ff.; GABRIEL/ HAUNGS/ZENDER 1984: 125 ff.).

Neben Aussagen über das Verhältnis von traditionellen und modernen Elementen enthalten einige dieser Studien detaillierte Informationen über die Einschätzung der Arbeit der kommunalen Vertretungskörperschaft, über die Beziehungen zwischen dem Rat und der Verwaltung und über die lokale Machtstruktur (vgl. ARZBERGER 1980: 106 ff.; GABRIEL/HAUNGS/ZENDER 1984: 161 ff.; SIMON 1988: 40 ff.). Beim Vergleich dieser Befunde ist allerdings zu berücksichtigen, daß in der Studie von GABRIEL/HAUNGS/ZENDER ausschließlich Angehörige der Minderheitsfraktion großstädtischer Vertretungskörperschaften vertreten waren. Deren Position im kommunalen Machtgefüge vermittelt möglicherweise ein verzerrtes Bild von der Realität kommunaler Demokratie.

Die Arbeit der Kommunalvertretung und deren Beziehung zur Verwaltung beurteilten die kommunalen Oppositionspolitiker aus Nordrhein-Westfalen ausgesprochen ambivalent: Die Hälfte von ihnen schätzte die Arbeit der kommunalen Vertretungskörperschaft positiv ein (46\%), die andere negativ (51\%). Die positiven Einschätzungen variierten in den einzelnen Städten zwischen 8 und 70\%, was auf beträchtliche Gemeindespezifika in den Machtstrukturen und in der lokalen Elitekultur hindeutet. Die Möglichkeiten des Rates, die Festlegung kommunalpolitischer Ziele in der betreffenden Stadt zu beeinflussen, wurde wesentlich positiver eingeschätzt als die Kontrolle der Verwaltung durch die Kommmunalvertretung (71\% bzw. $20 \%$ positive Angaben). Mängel in der Verwaltungskontrolle sahen die Ratsmitglieder eher bei der Vorbereitung (18\%) als beim Vollzug von Entscheidungen durch die Verwaltung (40\%), obgleich beide Aspekte mehrheitlich negativ eingeschätzt wurden.

$\mathrm{Da} B$ die Angaben der Oppositionspolitiker aus Nordrhein-Westfalen die Position der Minderheitsfraktion im kommunalen Entscheidungsprozeß $B$ reflektieren, zeigt sich bei einer Gegenüberstellung der Befunde von GABRIEL/HAUNGS/ZENDER (1984) mit denen SIMONs (1988). Die von SIMON befragten Ratsmitglieder und Angehörigen der Verwaltungsführung gehörten der Mehrheits- und der Minderheitspartei der Kommunalvertretung an. Ihre Aussagen vermitteln eine ausgewogenere Gewichtsverteilung zwischen der Kommunalvertretung und der Verwaltung als sie aus den Aussagen der nordrhein-westfälischen Oppositionspolitiker hervorgeht. Nach SIMON sahen die Ratsmitglieder die Initiative im kommunalen Entscheidungsprozeß eher bei den Ratsfraktionen (57\%) als bei der Verwaltung (16\%). Sie schrieben der Beratung von Vorlagen im Rat, in den Ausschüssen und in den Fraktionen eine substantielle Bedeutung für den Inhalt kommunalpolitischer Entscheidungen zu und stellten die in der Literatur verbreitete "Vorentscheider-These" in Frage. Auch die Möglichkeit des Rates, die Verwaltung zu kontrollieren, wurde erheblich positiver eingeschätzt als in der Untersuchung von GABRIEL/HAUNGS/ZENDER: Nur 29\% der Ratsmitglieder waren der Auffassung, der Rat könne die Verwaltung wegen Überlastung (11\%) bzw. wegen deren Abschirmungsstrategie (18\%) nicht kontrollieren, 53\% waren der Auffassung, der Rat sei zur Ausübung der Verwaltungskontrolle in der Lage, wenn er es wolle, weitere $18 \%$ schrieben ihm sogar uneingeschränkte Kontrollfunktionen zu. Die führenden Verwaltungsbeamten teilten diese Einschätzung. In Übereinstimmung mit den normativen Vorgaben der Gemeindeordnungen fand SiMON (1988: 40 ff., bes. 46) "kaum eine Stütze für die These ..., der Rat sei gegenüber der Verwaltung ohnmächtig." 
In der Untersuchung von ARZBERGER (1980: $59 \mathrm{ff} ., 106 \mathrm{ff}$.) stuften die Mitglieder der kommunalen Elite das Stadtparlament sogar überwiegend als die einflußreichste kommunalpolitische Institution ein. Abweichend von diesem Muster schrieben die Frankfurter Eliten der Stadtverwaltung den größeren Einfluß zu. Ob man diese Konstellation als Beleg für ein Machtgleichgewicht oder für eine Dominanz der lokalen Exekutive ansieht, hängt im wesentlichen davon ab, wie man die auf die Verwaltung und den Oberbürgermeister entfallenden Antworten interpretiert. Faßt man sie zusammen, dann zeichnen die Befragten in allen fünf Städten das Bild einer "exekutiven Führerschaft" (KEVENHÖRSTER 1977). Behandelt man sie getrennt, dann ist es angemessener, von einer Machtstreuung zu sprechen. Eine generelle Aussage über die Adäquanz der einen oder der anderen Interpretation ist jedoch kaum möglich, da die Koalitionsstrukturen im kommunalen Führungszentrum gemeinde- und politikfeldspezifisch variieren dürften. In Anbetracht der unzulänglichen Datenlage sind die Ursachen für diese widersprüchlichen Forschungsbefunde kaum auszumachen. Noch weniger ist eine generelle Aussage darüber möglich, wie die Bevölkerung die Gewichtsverteilung zwischen der Kommunalvertretung und der Verwaltung einschätzt.

Nach den bisher präsentierten Daten reflektieren die Einstellungen zur Kommunalpolitik zu einem erheblichen Teil generelle, für alle Ebenen des politischen Systems maßgebliche Wahrnehmungen und Bewertungen. Die noch in den fünfziger Jahren bestehenden Unterschiede zwischen der lokalen und der nationalen Politischen Kultur scheinen sich abgeschwächt zu haben. Nicht nur in struktureller, sondern auch in kultureller Hinsicht wurde die kommunale Selbstverwaltung mittlerweile in das politische System integriert. Sowohl die Bevölkerungsbefragungen als auch die Elitestudien vermitteln den Eindruck, daß die Kommunalpolitik nicht ausschließlich als sachrationaler Verwaltungsvollzug interpretiert wird. Parteienstaatlich-wettbewerbsdemokratische Vorstellungsmuster konnten sich auch auf der kommunalen Ebene etablieren. SIMON (1988: 102) bringt die einschlägigen Befunde zu dieser "Grunddimension Politischer Kultur in der Kommunalpolitik" zutreffend auf den folgenden Nenner:

"Die administrative Vorstellung von Kommunalpolitik überwiegt. Sie wird aber keineswegs so ausschließlich vertreten, wie gelegentlich unterstellt wird ... Vielmehr gibt eine beachtliche Minderheit einem politischen Verständnis der Kommunalpolitik den Vorzug. Beide Grundeinstellungen sind aber in der Regel nicht in einem reinen Typ vorzufinden, sondern in wechselnder Akzentuierung und Vermischung auch mit Elementen der jeweils anderen Einstellung. Dies ist unter anderem darauf zurückzuführen, daß die wenigsten Kommunalpolitiker solche Fragen in den Zusammenhang einer reflektierten Systemvorstellung bringen, sondern eher ad hoc und aufgrund von persönlichen Erfahrungen einzeln bewerten" (Hervorh. OWG).

\subsection{Partizipative Orientierungen: Politische Kompetenz und Handlungsbereitschaft}

$\mathrm{Zu}$ den interessanten Merkmalen der lokalen Politischen Kultur der Bundesrepublik gehören die partizipativen Orientierungen. Wie die Einstellungen zur Exekutive sind auch sie von den politischen Traditionen Deutschlands geprägt. Als Folge dieses Einflusses schätzten BAKER/DALTON/HILDEBRANDT (1981: 28) das staatsbürgerliche KompetenzbewuBtsein in der Gründungsphase der Bundesrepublik als "alarmierend niedrig" ein. GREIFFENHAGEN/GREIFFENHAGEN (1981: 67) charakterisierten die zu 
dieser Zeit vorherrschende Interpretation der staatsbürgerlichen Beteiligungsrechte wie folgt:

"die Bürger kommen ihrer Wahl'pflicht' nach, wie sie ihrer Steuerpflicht, ihrer Impfpflicht oder Schulpflicht nachkommen, als einer staatlichen Anordnung, der man Folge leisten $m u B^{\prime \prime}$.

Die schwache Verankerung demokratisch-partizipativer Normen im Nachkriegsdeutschland ist durch die empirische Forschung verhältnismäßig gut dokumentiert (vgl. GABRIEL 1986: 195 ff.). In Anbetracht der frühzeitigen Institutionalisierung politischer Mitwirkungsrechte auf der kommunalen Ebene stellt sich allerdings die Frage, ob dieser für die nationale Politische Kultur der Bundesrepublik charakteristische Zug auch das Verhältnis der Bevölkerung zur Kommunalpolitik bestimmt. Für eine Sonderstellung der partizipativen Elemente in der lokalen Politischen Kultur könnte neben der langen Selbstverwaltungstradition der Umstand sprechen, daß die Kommunalpolitik in Deutschland traditionell als Verwaltungshandeln angesehen wurden und daß die Bundesbürger sich im Umgang mit der Verwaltung schon in den fünfziger Jahren wesentlich selbstbewußter gaben als in der Einschätzung ihrer politischen Einflußmöglichkeiten (vgl. ausführlich: ALMOND/VERBA 1965: 136 ff.).

Kein anderer Aspekt der lokalen Politischen Kultur ist datenmäßig so gut erschlossen wie die partizipativen Orientierungen. In der Civic-Culture-Studie von 1959/60 erfaßten ALMOND/VERBA (1965: 136 ff.) diese Dimension der Politischen Kultur über das Konzept der subjektiven politischen Kompetenz. Die entsprechenden Fragen wurden 1974 und 1980 erneut in bundesweite Repräsentativerhebungen aufgenommen. Damit besteht die Möglichkeit, dieses wichtige Merkmal der lokalen Politischen Kultur in längerfristiger Perspektive zu untersuchen und dabei Veränderungen im Verhältnis der Bevölkerung zur Politik aufzuzeigen.

Als subjektive politische Kompetenz bezeichneten ALMOND/VERBA die Erwartung, man könne in politischen Konfliktsituationen Einfluß auf die politischen Entscheidungsträger ausüben. Für die auf die lokale Ebene gerichteten Einflußversuche prägten sie den Begriff der lokalpolitischen Kompetenz, die Wahrnehmung entsprechender Chancen in der nationalen Politik definierten sie als politische Kompetenz in nationalen Fragen. Ein Vergleich zwischen diesen beiden Einstellungen kann unter zwei Gesichtspunkten erfolgen: zunächst im Hinblick darauf, ob die Bürger im Bedarfsfalle überhaupt Möglichkeiten zur Einflußnahme sehen und weiterhin im Hinblick auf die in dieser Situation bevorzugten Einflußstrategien.

Wie ALMOND/VERBA ausführlich darlegten, bestand am Ende der fünfziger Jahre ein beträchtliches Gefälle zwischen der subjektiven Kompetenz der Bundesbürger in lokalen und nationalen Fragen. Während sich fast zwei Drittel der Befragten als lokalpolitisch kompetent einstuften, glaubten nicht einmal vier von zehn Personen, etwas gegen unerwünschte gesetzgeberische Vorhaben des Bundestages unternehmen zu können (vgl. Tabelle 8). Für die meisten von ihnen stellte sich die staatliche Politik tatsächlich als "Große Politik" dar, die sich weitgehend ihrem Zugriff entzog. Nicht zuletzt wegen dieser schwachen Ausprägung partizipativer Orientierungen bezeichneten ALMOND/VERBA (1965: 312 f.) die Politische Kultur der Bundesrepublik als Untertanenkultur. Allerdings traf diese Charakterisierung schon zum damaligen Zeitpunkt nur teilweise zu. Auf der lokalen Ebene manifestierte sich die lange Tradition kommunaler Selbstverwaltung nämlich in einem politischen Selbstbewußtsein der Bürger, das nicht wesentlich schwächer ausgeprägt war als in traditionsreichen De- 
mokratien (vgl. hierzu ALMOND/VERBA 1965: 136 ff.; GABRIEL 1990a: 20 ff.). In einer Fallstudie über das staatsbürgerliche Engagement der Stuttgarter Bevölkerung stellte WARREN (1957: 427) ein Nebeneinander obrigkeitsstaatlicher und partizipatorischer Elemente fest und kam somit zu einer ähnlichen Grundaussage wie ALMOND/ VERBA.

Tabelle 8: Subjektive Kompetenz in der lokalen und nationalen Politik in der Bundesrepublik Deutschland, 1959 - 1980 (Angaben: Prozentanteile).

"Angenommen, hier in der Gemeindeverwaltung (Stadtverwaltung) würde eine Verordnung in Betracht gezogen, die Sie als sehr ungerecht oder nachteilig betrachten. Was meinen Sie, könnten Sie dagegen tun?

Und wenn wir jetzt einmal annehmen, daß im Bundestag in Bonn ein Gesetz in Erwägung gezogen würde, das Sie als sehr ungerecht und nachteilig ansehen. Was meinen Sie, könnten Sie dagegen unternehmen?"

Einwohnerzahl der Gemeinde

\begin{tabular}{|c|r|r|r|r|}
\hline bis & 5.000 & 20.000 & 100.000 & Alle \\
5.000 & -19.999 & -99.999 & u.m. & Befragten \\
\hline
\end{tabular}

national

\begin{tabular}{|l|l|l|l|l|l|}
\hline 1959 & 31 & 36 & 36 & 49 & 38 \\
1974 & 50 & 51 & 56 & 64 & 56 \\
& 48 & 49 & 55 & 52 & 51 \\
\hline
\end{tabular}

lokal

\begin{tabular}{|l|l|l|l|l|l|}
\hline 1959 & 62 & 57 & 62 & 65 & 62 \\
1974 & 70 & 64 & 62 & 69 & 67 \\
1980 & 63 & 62 & 67 & 69 & 66 \\
\hline
\end{tabular}

Prozentpunktdifferenz lokal-national

\begin{tabular}{|c|c|c|c|c|c|}
\hline $\begin{array}{l}1959 \\
1974 \\
1980\end{array}$ & $\begin{array}{l}31 \\
20 \\
15\end{array}$ & $\begin{array}{l}21 \\
13 \\
13\end{array}$ & $\begin{array}{r}26 \\
6 \\
12\end{array}$ & $\begin{array}{r}16 \\
5 \\
17\end{array}$ & $\begin{array}{l}24 \\
11 \\
15\end{array}$ \\
\hline \multicolumn{6}{|l|}{$N$} \\
\hline $\begin{array}{l}1959 \\
1974 \\
1980\end{array}$ & $\begin{array}{l}354 \\
659 \\
166\end{array}$ & $\begin{array}{l}180 \\
427 \\
221\end{array}$ & $\begin{array}{l}149 \\
400 \\
247\end{array}$ & $\begin{array}{l}272 \\
757 \\
278\end{array}$ & $\begin{array}{r}955 \\
2243 \\
912\end{array}$ \\
\hline
\end{tabular}

Quellen: ALmond/VerBa 1965; Political Action I, Political Action II; Auswertung d. Verf.; 1974 blieben die Befragten unter 18 Jahren unberücksichtigt.

Da die lokalpolitische Kompetenz in der Bundesrepublik bereits 1959 verhältnismäßig stark ausgeprägt war, ist es nicht weiter erstaunlich, daß ihr Anstieg zwischen 1959 und 1974 wesentlich schwächer ausfiel als der Wandel der betreffenden Einstellungen zur nationalen Politik. Der Anteil lokalpolitisch Kompetenter lag 1974 nur um fünf Prozent über dem Niveau des Jahres 1959, der für die nationale Politik ermittelte Zuwachs betrug demgegenüber 18\%. Die zu Recht als "partizipatorische Revolu- 
tion" bezeichnete Ausbreitung partizipativer Einstellungen in der Bundesrepublik manifestierte sich vor allem in der nationalen Politik, in der in dieser Hinsicht auch ein besonders großer Nachholbedarf bestand. Wie der Vergleich mit den Daten des Jahres 1980 zeigt, war die Verstärkung der partizipativen Aspekte der Politischen Kultur der Bundesrepublik in der Mitte der siebziger Jahre weitgehend abgeschlossen. Der folgende Zeitraum zeichnete sich eher durch Kontinuität als durch politischen Wandel aus. Zwischen 1974 und 1980 blieb das lokalpolitische Kompetenzbewußtsein praktisch stabil (-1\%), im Hinblick auf die nationale Politik nahm die Perzeption staatsbürgerlicher Einflußchancen geringfügig ab (-5\%). In Folge dieser Entwicklung sah die Mehrheit der Bundesbürger 1974 und 1980 auf der lokalen und auf der nationalen Ebene die Möglichkeit, sich gegen ungerechtfertigte Aktivitäten der politischen Führung zur Wehr zu setzen, 1959 war dies nur in der Lokalpolitik der Fall gewesen.

Trotz dieser deutlichen Veränderung schwächte sich das Gefälle zwischen der Kompetenz in lokalen und nationalen Fragen nur graduell ab (1959: 24\%; 1980: $15 \%)$, es blieb ein stabiles Merkmal der Politischen Kultur der Bundesrepublik. Dies bestätigt auch eine Umfrage aus dem Jahre 1985, in der die Bevölkerung ihre Einflußchancen in der lokalen Politik deutlich höher einschätzte als auf der nationalen Ebene des politischen Systems. Während $29 \%$ der Bundesbürger der Aussage zustimmten "Der Normalbürger hat großen Einfluß auf die Kommunalpolitik", sahen nur 19\% in der Politik im allgemeinen entsprechende Einflußchancen. Für diese unterschiedlichen Einschätzungen dürften neben den organisatorischen Eigenarten der kommunalen bzw. nationalen Politik deren besondere politische Traditionen verantwortlich sein. Darüber hinaus ist zu vermuten, daß der starke Anstieg des nationalen Kompetenzbewußtseins in den Jahren 1959 bis 1974 durch partizipative Erfahrungen auf der lokalen Ebene, z.B. durch die Mitarbeit in Bürgerinitiativen, die Beteiligung an kommunalen Planungen oder die Mitarbeit in lokalpolitischen Organisationen, begünstigt wurde. In der lokalen Politik machte die Bevölkerung Erfahrungen erfolgreicher politischer Einflußnahme, die zu einer generellen Stärkung ihres staatsbürgerlichen Selbstbewußtseins führten (vgl. ähnliche Informationen in den Fallstudien von BIEGE et al. 1978: $155 \mathrm{f}$. und BUSE/NELLES/OPPERMANN 1978: $131 \mathrm{f} . ;$ anders: HOLTMANN/ KILLISCH 1989: 29 f.).

Neben der grundsätzlichen Möglichkeit, im Bedarfsfalle etwas gegen unerwünschte Maßnahmen der politischen Führung unternehmen zu können, beschrieben ALMOND und VERBA (1965: 145 ff.) die Präferenz für bestimmte Formen politischer Einflußnahme als einen Indikator staatsbürgerlich-partizipativer Einstellungen. In der Bereitschaft, zur Durchsetzung der eigenen Vorstellungen mit anderen zusammenzuarbeiten, sahen sie einen Hinweis auf interpersonales Vertrauen und eine positive Beziehung zur politischen Gemeinschaft. Auch an der Bereitschaft zur Verletzung demokratischer Spielregeln läßt sich die Einstellung zu demokratischen Werten und Normen ablesen: Die Neigung, zur Durchsetzung politischer Ziele auf gewaltsame oder illegale Mittel zurückzugreifen, deutet auf ein gebrochenes Verhältnis zu demokratisch-rechtsstaatlichen Prinzipien hin.

Im Hinblick auf die demokratietheoretische Bedeutung der Strategien politischer Einflußnahme konzentriert sich die folgende Untersuchung auf das Verhältnis zwischen individuellen und kollektiven Einflußstrategien, formalisierten bzw. informellen Formen der Einflußnahme sowie auf die Verbreitung politischer Gewalt und gewaltfreier Protestaktionen. 
Relativ knapp läßt sich das Problem der Gewaltanwendung in der lokalen und nationalen Politik abhandeln. Zu keinem Zeitpunkt gab es in der Bundesrepublik ein quantitativ bemerkenswertes Gewaltpotential. Die in der Literatur vielfach beklagten Anomietendenzen lassen sich durch die verfügbaren empirischen Daten nicht belegen (vgl. auch GABRIEL 1990b: 37 f., mit weiterer Literatur).

Tabelle 9: Bevorzugte Form der Einflußnahme auf die lokale und nationale Politik in der Bundesrepublik Deutschland, 1959-1980

(Angaben: Prozentanteile).

\begin{tabular}{|l|c|c|c|c|c|c|}
\multicolumn{1}{c}{} & \multicolumn{3}{c|}{ Lokal } & \multicolumn{3}{c|}{ National } \\
\cline { 2 - 7 } \multicolumn{1}{l|}{} & 1959 & 1974 & 1980 & 1959 & 1974 & 1980 \\
\hline Informelle Gruppen & 13 & 20 & 12 & 7 & 9 & 7 \\
Partei & 3 & 2 & 1 & 6 & 4 & 3 \\
Interessenverband & 5 & 1 & 7 & 6 & 1 & 4 \\
Indiv. Politikerkontakt & 13 & 11 & 13 & 10 & 13 & 13 \\
Indiv. Behördenkontakt & 26 & 9 & 8 & 4 & 1 & 1 \\
Rechtsweg & 1 & 1 & 2 & 0 & 1 & 1 \\
Öffentlichkeit mobilisieren & $\mathrm{x}$ & 4 & 3 & $\mathrm{x}$ & 2 & 1 \\
Wählen, Abstimmen,Kandidieren & 1 & 3 & 3 & 3 & 13 & 10 \\
Gewalt & 1 & 0 & 0 & 1 & 0 & 0 \\
Gewaltfreier Protest & $\mathrm{x}$ & 12 & 10 & $\mathrm{x}$ & 11 & 8 \\
Protest, unspezifisch & $\mathrm{x}$ & 0 & 7 & $\mathrm{x}$ & 0 & 2 \\
Sonstiges & 0 & 5 & 2 & 0 & 2 & 1 \\
Nichts & 31 & 29 & 30 & 56 & 39 & 43 \\
k.A. & 7 & 4 & 5 & 7 & 5 & 6 \\
\hline N & 955 & 2243 & 912 & 955 & 2243 & 912 \\
\hline
\end{tabular}

Quellen und Fragen wie in Tabelle 8; $x=$ nicht erhoben.

Unter den systemkonformen Instrumenten kommunalpolitischer Einflußnahme setzte die Bevölkerung 1959 vornehmlich auf individuelle Aktivitäten und weniger auf kollektive Strategien. Vier von zehn Befragten nannten auf die entsprechende Frage an erster Stelle individuelle Politiker- oder Behördenkontakte, das Beschreiten des Rechtsweges oder die Teilnahme an Wahlen und Abstimmungen. Nur jeder fünfte gab als die wichtigste Form kommunalpolitischer Einflußnahme die Mitarbeit in einer informellen Gruppe, einer Partei oder einem Interessenverband an. Formalisierte Wege der Einflußnahme waren erheblich weiter verbreitet als die Mobilisierung informeller Gruppen. Im Hinblick auf die politisch-kulturelle Tradition Deutschlands war der hohe Stellenwert individueller Verwaltungskontakte nicht überraschend, wohl aber die relativ häufige Nennung von Politikerkontakten. Beschränkt man die Auswertung auf diejenigen Befragten, die sich als lokalpolitisch kompetent einstuften, dann entfielen fast zwei Drittel aller Angaben auf Behörden- und Politikerkontakte. Daneben spielte nur noch die Mobilisierung informeller Gruppen eine gewisse Rolle. Alle übrigen Aktionsmöglichkeiten, danunter auch die Einflußnahme über politische Parteien und Interessenverbände, wurden nur von einer kleinen Minderheit der Befragten genannt. In der nationalen Politik standen individuelle Kontakte zu Politikern und Behördenvertretern ebenfalls im Vordergrund. Fast ebenso wichtig war die Ein- 
flußnahme über die politischen Parteien und die Interessenverbände, gefolgt von den informellen Gruppenaktivitäten. Allerdings wurden sämtliche Einflußstrategien infolge des niedrigen Niveaus nationaler politischer Kompetenz relativ selten genannt (vgl. Tabelle 9).

Wendet man sich dem Wandel in den Strategien kommunalpolitischer Interessendurchsetzung zu, dann fält vor allem der Bedeutungsverlust der Verwaltungskontakte auf. Während 1959 noch jeder vierte Befragte den Weg über die Verwaltung als besonders geeignetes Mittel einschätzte, seinen Interessen Geltung zu verschaffen, traf dies 1980 nur noch für acht Prozent zu. In keinem anderen Bereich waren annähernd so große Veränderungen zu verzeichnen. Dieses Ergebnis spricht für einen fundamentalen, auch für die nationale Politik typischen Wandel der Politischen Kultur: die abnehmende Bedeutung der Exekutive als Ansprechpartner der politisch Aktiven (vgl. auch GrEIFFENHAGEN/GrEIFFENHAGEN 1981: 85). Damit ging ein starker Bedeutungsgewinn lokaler Protestaktionen einher. 1959 waren sie noch nicht als eigenständige Formen der Einflußnahme erfaßt worden, 1980 nannten $17 \%$ aller Befragten (gewaltfreie) Protestaktivitäten als wichtigstes Mittel kommunalpolitischer Einflußnahme. Alle übrigen Einflußstrategien veränderten sich nur graduell oder diskontinuierlich. Dabei erscheint mir der durchgängig geringe Stellenwert der Parteien und Verbände als Einrichtungen kommunalpolitischer Partizipation bemerkenswert.

Ein Vergleich zwischen den Mustern nationaler und lokaler politischer Einflußnahme läßt deutliche Unterschiede in der Struktur und in der Entwicklung erkennen. Als das wichtigste Mittel zur Einflußnahme auf die nationale Politik schätzten die Bundesbürger $\mathrm{zu}$ allen Erhebungszeitpunkten individuelle Politikerkontakte ein. Der auf sie entfallende Prozentanteil war auBerordentlich stabil. Daneben wurden in erster Linie die Teilnahme an Wahlen, die Mobilisierung von Ad-hoc-Gruppen und Protestaktionen genannt. Wie auf der lokalen Ebene verzeichneten letztere den stärksten Zuwachs, die Parteien und Interessenverbände verloren demgegenüber auf niedrigem Niveau an Bedeutung. Die divergierende Entwicklung des lokalen und nationalen KompetenzbewuBtseins führte zwischen 1959 und 1980 zu einer Annäherung dieses Aspektes der lokalen Politischen Kultur an das nationale Muster - oder umgekehrt. Unterschiede bestehen nun vor allem im Niveau der Kompetenz, weniger in der Qualität der bevorzugten Einflußstrategien. Darüber hinaus indizieren die Veränderungen auf den verschiedenen Ebenen des politischen Systems einen gewissen Bruch mit der Tradition der Untertanenkultur, als deren wesentliche Bestandteile ALMOND und VERBA den Formalismus und die Exekutivorientierung der Bürger beschrieben hatten (vgl. ALMOND/VERBA 1965: 168 ff., bes. 182 ff.; vgl. auch GREIFFENHAGEN/ GREIFFENHAGEN 1981: 65 ff.).

Im Rahmen einer Analyse der lokalen Politischen Kultur sind die Angaben zum tatsächlichen Partizipationsverhalten, wie wir sie in einigen Fallstudien finden (vgl. ARZBERGER 1980: 115 ff., 127 ff.; BUSE/NELLES/OPPERMANN 1978: 226 ff.; HOLTMANN/KILLISCH 1989: 29 ff.; HILL 1987: 90 ff.; JARREN et al. 1989: 220 ff.; OEL 1970: 149 ff.; Hinweise auf ältere Untersuchungen bei GABRIEL 1979: 114 ff.), von untergeordneter Bedeutung. Aufmerksamkeit verdient dagegen ein in ARZBERGERS (1980: 119 ff.) Untersuchung enthaltener Aspekt dieses Problems, die Bewertung der Effektivität einzelner Partizipationsformen als Mittel kommunalpolitischer Einflußnahme. Diese Einstellungen ähneln thematisch der lokalpolitischen Kompetenz, allerdings ermittelte ARZBERGER - anders als ALMOND/VERBA und die an sie anknüpfenden Forscher - die perzipierte Wirksamkeit politischer Beteiligung durch eine ge- 
schlossene Frage. Die Angaben der Bevölkerung der fünf Städte über die geeigneten Einflußformen fielen insgesamt recht unterschiedlich aus, in einigen Punkten jedoch bestanden Übereinstimmungen in der Bewertung: Eine besonders wichtige Form der kommunalpolitischen Einflußnahme sah man überall in der Stimmabgabe bei Kommunalwahlen. Sie rangierte in zwei Städten auf dem ersten, in den drei anderen auf dem zweiten Platz. Eine vergleichbar große Bedeutung kam nur noch den Kontakten zum Oberbürgermeister zu, die sich in drei Kleinstädten auf dem ersten und in einer weiteren auf dem zweiten Platz befand. Nur in Frankfurt schätzte man diese Einflußstrategie etwas kritischer ein. Schließlich stuften in allen fünf Städten nur sehr wenige Befragte unkonventionelle Formen politischer Einflußnahme als besonders effektiv ein. Im Hinblick auf die große Bedeutung formalisierter Einflußstrategien stimmt dieser Befund mit den bei der Analyse der subjektiven politischen Kompetenz festgestellten Ergebnissen überein. Bezüglich der Bedeutsamkeit von Protestaktionen weichen die betreffenden Untersuchungsergebnisse erheblich voneinander $a b$.

Weder 1959 noch 1980 bestand zwischen der politischen Kompetenz und der Gemeindegröße eine statistisch signifikante oder substantiell interpretierbare Beziehung. Das Kompetenzgefälle zwischen der lokalen und der nationalen Politik fand sich in kleinen Gemeinden, Klein-, Mittel- und Großstädten. 1959 nahm das Kompetenzgefälle mit zunehmender Gemeindegröße ab, 1980 dagegen war ein derartiges Muster nicht mehr zu erkennen. Demnach gehört es zu den generellen Merkmalen der Politischen Kultur der Bundesrepublik, daß sich die Bevölkerung eher zur Einflußnahme auf die Kommunalpolitik in der Lage fühlt als zur Beeinflussung der nationalen Politik (vgl. Tabelle 8).

\subsection{Differenzierungsmerkmale: Gibt es lokale politische Subkulturen?}

Mit dem Stadt-Land-Unterschied ist das für unsere Untersuchung wichtigste Differenzierungsmerkmal Politischer Kultur angesprochen. Dies schließt natürlich nicht aus, daß weitere, bei einer globalen Betrachtung eines repräsentativen Bevölkerungsquerschnitts verdeckte Faktoren die Einstellungen zur lokalen Politik prägen. Derartige Differenzierungsprozesse bezeichnet man als Subkulturbildung. Subkulturen können sich vor allem herausbilden auf der Grundlage von

- Zugehörigkeit zu bestimmten sozialen Gruppen (Indikator: Konfessions- und Schichtzugehörigkeit),

- Sozialisation (Indikator: Bildungsniveau),

- Alter und

- Geschlecht.

Schließlich können ideologische Dispositionen, etwa die Selbsteinstufung auf dem Links-Rechts-Kontinuum, materialistische/postmaterialistische Wertorientierungen oder Parteiloyalitäten die Einstellungen zu politischen Objekten beeinflussen.

Im Rahmen dieser Untersuchung ist es weder möglich noch erforderlich, das gesamte Spektrum möglicher Bestimmungsfaktoren der lokalen Politischen Kultur zu erörtern. Es soll lediglich knapp skizziert werden, in welchen sozio-politischen Gruppen charakteristische, vom Durchschnitt der Befragten deutlich abweichende Einstellungen zur Kommunalpolitik auftreten. Dariber hinaus wird die Analyse auf einige besonders wichtige kommunalpolitische Einstellungen begrenzt. Unter den Ge- 
sichtspunkten der theoretischen Relevanz und der Verfügbarkeit aktueller Daten beschränkt sich die weitere Untersuchung auf die Gemeindeidentifikation, auf die Einstellungen zu den Parteien und zur Kommunalverwaltung sowie auf die lokalpolitische Kompetenz.

Wie aus Abbildung 2 hervorgeht, bilden die lokalpolitische Kompetenz und die Gemeindeidentifikation ein Gegensatzpaar. Befragtengruppen, in denen das eine Merkmal überdurchschnittlich weit verbreitet ist, weisen das andere nur in unterdurchschnittlichem Ausmaß auf. Ein besonders stark ausgeprägtes lokalpolitisches Kompetenzbewußtsein finden wir bei den Angehörigen höherer Statusgnuppen (hohes formales Bildungsniveau, hohes Einkommen, Selbstzuordnung zur oberen Mittelschicht/Oberschicht), bei Personen mittleren Alters sowie bei Befragten mit postmaterialistischen Wertorientierungen und schwachen religiösen Bindungen. Postmaterialistische Wertorientierungen, die Zugehörigkeit zur Neuen Mittelschicht und ein hohes formales Bildungsniveau zeichnen auch Personen mit einer unterdurchschnittlichen Gemeindeidentifikation aus. Dagegen fühlen sich Materialisten, Bewohner von Kleinstgemeinden, Befragte mit einem niedrigen Einkommen und Personen ohne parteipolitisch-ideologische Bindungen ihrer Gemeinde in besonderem Maße verbunden.

Abbildung 2: Gruppenspezifische Einstellungen zur Kommunalpolitik

\begin{tabular}{|c|c|c|}
\hline & überdurchschnittlich & unterdurchschnittlich \\
\hline $\begin{array}{l}\text { Gemeinde- } \\
\text { identifikation }\end{array}$ & $\begin{array}{l}\text { - keine Partei- } \\
\text { präferenz } \\
\text { - Materialisten } \\
\text { - niedriges Ein- } \\
\text { kommen } \\
\text { - Kleinstgemeinde }\end{array}$ & $\begin{array}{l}\text { - Postmaterialisten } \\
\text { - Neue Mittelschicht } \\
\text { - hohes Bildungs- } \\
\text { niveau }\end{array}$ \\
\hline $\begin{array}{l}\text { positive Einstellung } \\
\text { zu Parteien in der } \\
\text { Kommunalpolitik }\end{array}$ & $\begin{array}{l}\text { - SPD-Wähler } \\
\text { - linke Ideologie } \\
\text { - Großstadt }\end{array}$ & \\
\hline $\begin{array}{l}\text { Zufriedenheit mit der } \\
\text { Kommunalverwaltung }\end{array}$ & & $\begin{array}{l}\text { - GRÜNEN-Wähler } \\
\text { - keine Parteipräferenz }\end{array}$ \\
\hline $\begin{array}{l}\text { lokalpolitische } \\
\text { Kompetenz }\end{array}$ & $\begin{array}{l}\text { - Postmaterialisten } \\
\text { - hohes Bildungs- } \\
\text { niveau } \\
\text { - hohes Einkommen } \\
\text { - Obere Mittel- } \\
\text { - schich /Oberschicht } \\
\text { - mittleres Alter } \\
\text { - keine/schwache } \\
\text { relig. Bindung }\end{array}$ & $\begin{array}{l}\text { - keine Partei- } \\
\text { bindung } \\
\text { - Materialisten } \\
\text { rechte Ideologie }\end{array}$ \\
\hline
\end{tabular}

Weniger deutlich unterscheiden sich die Einstellungen bestimmter Bevölkerungsgruppen zu den Lokalparteien und zur Kommunalverwaltung. Keine Teilgruppe ist 
mit der Kommunalverwaltung überdurchschnittlich zufrieden, besonders verwaltungskritisch geben sich die Wähler der Grünen und parteipolitisch ungebundene Personen. Als Befürworter einer parteipolitisch orientierten Kommunalpolitik erweisen sich Großstädter, SPD-Wähler und Personen, die ihren ideologischen Standort als links einstufen.

Da die Zusammenhänge zwischen den Hintergrundvariablen und den politischen Einstellungen im allgemeinen schwach ausgeprägt sind, lassen sich diese Befunde kaum als Hinweise auf die Ausbildung spezifischer lokaler Subkulturen bewerten. In den meisten Fällen unterscheidet sich lediglich das Ausmaß, in dem bestimmte Einstellungen in den Vergleichsgruppen auftreten, nicht aber ihre generelle Ausrichtung. Aus diesem Grunde sollte man sich vor einer Überinterpretation der beschriebenen Unterschiede hüten. Dies gilt insbesondere für die Einstellungen zu den politischen Parteien und zur Verwaltung, die in der traditionellen Selbstverwaltungsdoktrin als Gegenpole im politischen System der Gemeinde angesehen wurden. Die Gemeinsamkeiten in der Bewertung überwiegen bei weitem die Unterschiede. Im Ansatz läßt sich ein gewisser Anti-Institutionalismus unter den Anhängern der Neuen Linken erkennen.

Etwas eindeutiger stellen sich die Interpretationsmöglichkeiten für die zur Gemeindeidentifikation und zur lokalpolitischen Kompetenz vorgelegten Befunde dar. Eine starke lokale Bindung und ein schwaches lokalpolitisches Kompetenzbewußtsein findet man vor allem in solchen gesellschaftlichen Gruppen, die sich durch einen niedrigen sozialen Status, traditionelle politische Wertvorstellungen und eine schwache Parteibindung auszeichnen. Die aus der Partizipationsforschung bekannten Erkenntnisse über die Struktur- und Einstellungskorrelate politischer Partizipation decken sich mit unseren Daten über die Bestimmungsfaktoren lokalpolitischer Kompetenz (vgl. z.B. Milbrath/GoEL 1977). Auch auf dem Hintergrund gesellschaftlicher Modernisierungstheorien werden die beschriebenen Zusammenhänge leicht nachvollziehbar.

Personen mit einem stark entwickelten Kompetenzbewußtsein und einer schwach ausgeprägten Gemeindebindung gehören typischerweise den Trägergruppen der sich entfaltenden nachindustriellen Gesellschaft an. Dieser Tatbestand weist sie als Repräsentanten einer Konzeption kommunaler Selbstverwaltung aus, in der partizipative und kosmopolitische Vorstellungen eine besondere Rolle spielen. Besonders klar zeigen sich diese Konturen einer neuen lokalen Politischen Kultur in den Großstädten, in denen die Differenzierung der kommunalpolitischen Einstellungen an der Materialismus-Postmaterialismus-Konfliktlinie deutlicher ausgeprägt ist als anderswo. Vor allem hier gibt es Ansätze zur Ausbildung einer besonderen, postmaterialistisch-alternativen Subkultur. Sie bildet den Nährboden für die Wahlerfolge der GRÜNEN. Die großstädtisch-alternative Subkultur umfaßt zwar nur einen relativ kleinen Teil der Bevölkerung, dieser ist aber in besonderem Maße politisiert und aktionsbereit.

\section{Übungsfrage 4:}

a) Gibt es grundsätzliche Unterschiede zwischen lokaler und nationaler Politischer Kultur?

b) Ist ein entscheidender Einfluß der Gemeindegröße auf die Ausprägung der lokalen Politischen Kultur festzustellen? 


\section{Zusammenfassung und Schlußfolgerungen}

\subsection{Charakteristika der lokalen Politischen Kultur der Bundesrepublik}

Aus den bislang präsentierten Analysen lassen sich einige allgemeine Aussagen über die Eigenschaften der lokalen Politischen Kultur der Bundesrepublik ableiten:

O Die einschneidenden gesellschaftlichen Veränderungen und die weitreichende Neuordnung des kommunalen Gebietsbestandes haben die Integrationsfunktion der Kommunen nicht beseitigt, aber doch deutlich geschwächt. Noch in der Mitte der siebziger Jahre war die Bindung der Bürgerinnen und Bürger an ihre Gemeinde stärker als die Verbundenheit mit der Nation. Dieser Sachverhalt verdient vor allem deshalb Erwähnung, weil sich in den dreißig Jahren nach der Gründung der Bundesrepublik ein nachhaltiger Wandel der nationalen Politischen Kultur vollzog, in dessen Verlauf viele traditionelle Orientierungen abgeschwächt wurden. Die Gemeindebindung war von dieser Entwicklung allenfalls graduell betroffen.

O Die Daten zum Interesse an der Kommunalpolitik, zum subjektiven Verständnis kommunalpolitischer Fragen und zur lokalpolitischen Kompetenz stützen die These, die kommunale Selbstverwaltung zeichne sich im Vergleich mit der staatlichen Politik durch eine besondere Bürgernähe aus.

O Das für die deutsche Selbstverwaltungstradition typische Spannungsverhältnis zwischen Parteipolitik und angeblicher Verwaltungsrationalität besteht in der Vorstellungswelt der Bevölkerung und der politischen Führungsgnuppen der Gemeinden tendenziell fort. Politik und Verwaltung stellen sich den Bürgern und den Kommunalpolitikern aber nicht als unvereinbare Gegensätze dar. Im Einklang mit ihrer traditionell positiven Beziehung zur Exekutive äußert sich die Mehrheit der Bundesbürger zufrieden mit der Kommunalverwaltung, jedoch akzeptiert sie mittlerweile auch die Rolle der Parteien in der Kommunalpolitik.

Wegen der großen Lücken im Datenmaterial ist eine umfassende und tiefgehende Analyse nicht möglich. Dennoch gilt es festzuhalten, daß sich aus den ausgewerteten Materialien keine Hinweise auf besondere demokratisch-partizipative Defizite der lokalen Politischen Kultur der Bundesrepublik ergeben. Diese Feststellung gilt im internationalen Vergleich wie auch im Vergleich mit der nationalen Politischen Kultur. Die meisten Merkmale der lokalen Politischen Kultur finden wir in ähnlicher Form auch in den Einstellungen der Bundesbürger und -bürgerinnen zur nationalen Politik. Staat und Gemeinde bilden für sie eine Einheit. Die lokale Politische Kultur ist fest in der umfassenden nationalen Kultur verankert.

O Bei einem Vergleich von Gemeinden unterschiedlicher Größe müssen diese Aussagen allenfalls geringfügig modifiziert werden. Wenn man sich die beträchtichen Unterschiede in den politischen Strukturen von Landgemeinden, Klein-, Mittel- und Großstädten vergegenwärtigt, dann sind die Divergenzen in den politischen Einstellungen der Bewohner dieser Gemeinden erstaunlich schwach ausgeprägt. Eine Differenzierung der Politischen Kultur in eine ländliche, eine klein-, mittel- und eine großstädtische Subkultur läßt sich empirisch allenfalls in schwachen Ansätzen nachweisen. Lediglich die Bewohner kleiner Gemeinden setzen sich in ihren Einstellungen bis zu einem gewissen Grade von der übrigen Bevölkerung ab. Ihre Vorstellungen von Kommunalpolitik sind stärker traditionsverhaftet. 
Mangels brauchbarer Daten kann nicht entschieden werden, wodurch der relativ schwache Zusammenhang zwischen dem Gemeindetyp und den Einstellungen zur lokalen und nationalen Politik bedingt ist. Möglicherweise erfaßt der Indikator "Gemeindegröße" die wirklich relevanten Differenzierungsmerkmale nicht angemessen; denn die Lebensformen in einer 1.000-Seelen-Gemeinde in einem städtischen Verdichtungszentrum dürften sich von denen einer gleich großen Gemeinde in einer dünn besiedelten und strukturschwachen Region unterscheiden. Vorstellbar ist aber auch, daß der gesellschaftliche Modernisierungsproze $B$, in dem sich die gesamte westliche Welt seit dem Ende des Zweiten Weltkrieges befindet, die früher bestehenden Stadt-Land-Unterschiede zunehmend nivelliert hat.

\subsection{Lokale Politische Kultur und kommunale Demokratie - Zur systematischen Bedeutung politisch-kultureller Faktoren für die Funktionsweise des politischen Systems}

Zwei Faktoren erschweren die systematische Charakterisierung der vorherrschenden Merkmale der lokalen Politischen Kultur der Bundesrepublik: die unzulängliche $\mathrm{Da}$ tengrundlage und das Fehlen eindeutiger Aussagen über die für eine demokratische Politische Kultur charakteristische Verteilung politischer Einstellungen. Dennoch kann man die Bestandsaufnahme mit einer eindeutigen Feststellung eröffnen. Die Bürgerinnen und Bürger nehmen die lokale und die nationale Politik auf unterschiedliche Weise wahr. Weder die lokale noch die nationale Politische Kultur der Bundesrepublik läßt sich als homogen charakterisieren: Bei allen untersuchten Einstellungen wurde eine mehr oder weniger große Streuung festgestellt, die in einigen Fällen mit der Zugehörigkeit zu bestimmten sozio-politischen Gruppen variierte. Es wäre allerdings überzogen, darin eine Fragmentierung der Politischen Kultur zu sehen.

Wie sich aus ALMOND/VERBAS Unterscheidung zwischen unterstützenden, indifferenten und entfremdeten Politischen Kulturen ergibt, ist für die Charakterisierung einer Politischen Kultur das Verhältnis von positiven und negativen Einstellungen zur Politik bedeutsam. Zu einer funktionsfähigen Demokratie gehört eine unterstïtzende Politische Kultur. Diese immer wieder als "affirmativ" und "herrschaftsstabilisierend" kritisierte Feststellung (vgl. z.B. REICHEL 1981: 26 ff.) basiert auf der plausiblen Hypothese, daß kein politisches System auf Dauer ohne die Unterstützung oder zumindest die Duldung durch die Mehrheit seiner Bürgerinnen und Bürger funktionsfähig ist. Dies gilt erst recht für eine Demokratie. Obgleich ALMOND und VERBA keine Schwellenwerte angeben, jenseits derer sie eine Politische Kultur als unterstützend einstufen, dürfte dies umso stärker der Fall sein, je größer der Anteil positiver Einstellungen zum politischen System und zu einzelnen Elementen ausfällt. Für eine Präzisierung dieses noch immer reichlich unbestimmten Konzeptes bestehen zwei Möglichkeiten: Eine Politische Kultur läßt sich dann als unterstützend bezeichnen, wenn mehr als die Hälfte der Bürger das politische System und seine Elemente positiv bewertet oder wenn positive Einstellungen zur Politik häufiger auftreten als negative. In unserem Falle macht es nur bei der Einstellung zur kommunalpolitischen Rolle der Parteien einen Unterschied, welche dieser beiden Vorgehensweisen man wählt.

Klammert man die demokratietheoretisch kaum interpretierbare Frage nach der Gemeindeidentifikation aus der weiteren Betrachtung aus, dann verbleiben drei für eine systematische Darstellung der Eigenschaften der lokalen Politischen Kultur der Bundesrepublik verwertbare Indikatoren: die Einstellungen zur Rolle der Parteien in 
der Kommunalpolitik, die Zufriedenheit mit den Leistungen der Kommunalverwaltung und die lokalpolitische Kompetenz. In der spezifischen deutschen Selbstverwaltungstradition gehört die Einschätzung der kommunalpolitischen Position der Parteien zu den grundsätzlichen regimebezogenen Orientierungen. Im Spannungsfeld zwischen einer verwaltungsorientiert-harmonistischen und parteienstaatlich-konfliktorientierten Konzeption der Kommunalpolitik kommt den Parteien eine Schlüsselstellung zu. Die Einschätzung der Leistungen der Kommunalverwaltung dürfte aus den genannten Gründen in erster Linie die regulative Dimension lokaler Politik betreffen, allerdings könnten mit dieser Frage auch Regimeorientierungen angesprochen sein. Die lokalpolitische Kompetenz schließlich gehört zu den partizipativen Orientierungen. Damit sind die wichtigsten Beschreibungsdimensionen der lokalen Politischen Kultur, die System-, die Input- und die Output-Orientierungen, über mindestens einen Indikator abgedeckt.

Unter dem Gesichtspunkt des Überwiegens positiver gegenüber negativen Einstellungen stellt sich die lokale Politische Kultur der Bundesrepublik als unterstützend dar. Positive Einstellungen zu den Parteien und zur Kommunalverwaltung sind häufiger als negative Orientierungen auf die betreffenden Objekte: Obgleich die Verwaltung nach wie vor höheres Ansehen genieBt, wuchs auch die Akzeptanz der politischen Parteien. Positive Einstellungen zur Rolle der Parteien in der Kommunalpolitik waren in den siebziger Jahren weiter verbreitet als parteienkritische Vorstellungen. Dies spricht für die Möglichkeit einer Verbindung zwischen traditionellen und modernen Vorstellungen von kommunaler Selbstverwaltung.

Auch ein zweites für die nationale Politik der Bundesrepublik lange Zeit typisches Spannungsverhältnis scheint sich abgeschwächt zu haben: das Ungleichgewicht zwischen einem außerordentlich hohen Vertrauen in die Exekutive und einem schwachen staatsbürgerlichen Selbstbewußtsein. Bereits in "The Civic Culture" stellten ALMOND und VERBA (1965) fest, die Partizipantenrolle sei in der lokalen Politik weitaus stärker entwickelt als in der nationalen Politik. Wenn man die neueren Daten zur lokalpolitischen Kompetenz und zur Zufriedenheit mit der Kommunalverwaltung miteinander vergleicht, dann besteht das traditionelle Ungleichgewicht zwischen partizipativen und regulativen Orientierungen nicht mehr. Allerdings schwanken die in den einzelnen Umfragen enthaltenen Angaben über die Einflußmöglichkeiten der Bürger auf die lokale Politik so stark, daß generelle Aussagen zu diesem Problem nur unter großen Vorbehalten möglich sind.

Sofern die schwache Datenbasis überhaupt Verallgemeinerungen zuläßt, entspricht die lokale Politische Kultur der Bundesrepublik weitgehend den Anfordenungen an eine demokratische Staatsbürgerkultur: Die Mehrheit der Bevölkerung vertraut der Exekutive, und sie hält sich selbst für fähig, das Handeln der lokalen politischen Führung zu beeinflussen. Den politischen Parteien bringt sie mehr positive als negative Gefühle entgegen. Mit zunehmender Gemeindegröße schwächt sich das Vertrauen zur Exekutive graduell ab, die Einschätzung der politischen Parteien wird positiver und das Kompetenzbewußtsein der Bürgerinnen und Bürger steigt. Die Balance verschiebt sich also von einer traditionellen Untertanenorientierung zu einer partizipativen, konfliktorientierten Politischen Kultur.

Obgleich sich die nationale Politische Kultur der Bundesrepublik im Prinzip durch dieselben Merkmale auszeichnet, bestehen einige interessante und aufschlußreiche Unterschiede zwischen der Beziehung der Bevölkerung zur lokalen und zur nationalen Politik. Noch immer wird die Rolle der Parteien auf der Bundesebene etwas posi- 
tiver eingeschätzt als in den Kommunen. Zwar wäre es bei der gegebenen Datenlage verfehlt, von einem Antiparteienaffekt zu sprechen, seine Restbestände sind aber eher auf der lokalen Ebene anzutreffen als in der nationalen Politik. Dagegen ist das Kompetenzbewußtsein der Bevölkerung auf der lokalen Ebene deutlich stärker entwickelt als in der nationalen Politik. Insofern trifft die Einschätzung der Kommunalpolitik als einen besonders bürgernahen Bereich zu.

Nach dem Ergebnis unserer Untersuchung erweist sich die lokale Politische Kultur der Bundesrepublik als ein integrierter Bestandteil der nationalen Politischen Kultur. Die Einbindung der Kommunen in den demokratischen Staatsaufbau blieb nicht auf die formal-institutionelle Ebene beschränkt, sondern sie wurde offenkundig von der Bevölkerung angenommen. Hierfür spricht jedenfalls die relativ große Übereinstimmung in den Einstellungen zur nationalen und zur lokalen Politik.

Übungsaufgabe 5:

Formulieren Sie zwei oder drei Vermutungen zur Politischen Kultur Ihrer Gemeinde! Skizzieren Sie ein Verfahren, mit dem die Vermutungen geprüft werden könnten!

\section{Literatur}

ALmond, Gabriel A. 1956: Comparative Political Systems. In: Journal of Politics, 18. Jg., Heft 3, S. 391-409.

ALmOND, Gabriel A. 1980: The Intellectual History of the Civic Culture Concept. In: ALmond, Gabriel A./NERBA, Sidney (Hrsg.): The Civic Culture Revisited. An Analytic Study, S. 1-36, Boston.

ALMOND, Gabriel A. 1987: Politische Kulturforschung - Rückblick und Ausblick. In: BERG-SCHLOSSER, Dirk/SCHISSLER, Jakob (Hrsg.): Politische Kultur in Deutschland. Bilanz und Perspektiven der Forschung. Politische Vierteljahresschrift, Sonderheft 18, S. 27-38, Opladen.

Almond, Gabriel A./VERBA, Sidney 1965: The Civic Culture. Political Attitudes and Democracy in Five Nations, Boston.

Almond, Gabriel A./VERBA, Sidney (Hrsg.) 1980: The Civic Culture Revisited. An Analytic Study, Boston.

ARZBERGER, Klaus 1980: Bürger und Eliten in der Kommunalpolitik, Stuttgart et al. ARZBERGER, Klaus/MURCK, Michael/SchUMACHER, Jürgen 1979: Die Bürger. Bedürfnisse, Einstellungen, Verhalten, Königstein.

BAKER, Kendall L./DALTON, Russell J./HILDEBRANDT, Kai 1981: Germany Transformed. Political Culture and the New Politics, Cambridge, Mass./London.

BERG-SCHLOSSER, Dirk/SCHISSLER, Jakob (Hrsg.): Politische Kultur in Deutschland. Bilanz und Perspektiven der Forschung. Politische Vierteljahresschrift, Sonderheft 18, Opladen. 
BIEGE, Hans Peter et al. 1978: Zwischen Persönlichkeitswahl und Parteientscheidung. Kommunales Wahlverhalten im Lichte einer Oberbürgermeisterwahl, Königstein.

BRETSCHNEIDER, Michael/BICK, Wolfgang 1989a: Kommunale Umfrageforschung. Erfahrungsberichte aus zehn Städten, Berlin.

BRETSCHNEIDER, Michael/BICK, Wolfgang 1989b: Kommunale Bürgerbefragungen auf dem Wege zum Stadt- und Regionalvergleich. In: ZA-Information, 25. Jg., S. 9094.

BUSE, Michael J./NELLES, Wilfried/OPPERMANN, Thomas 1978: Determinanten politischer Partizipation. Theorieansatz und empirische Überprüfung am Beispiel der Stadt Andernach, Meisenheim am Glan.

CONRADT, David P. 1980: Changing German Political Culture. In: ALMOND, Gabriel A./VERBA, Sidney (Hrsg.): The Civic Culture Revisited. An Analytic Study, S. 212272, Boston.

DIAS, Patrick 1970: Der Begriff "Politische Kultur" in der Politikwissenschaft. In: OBERNDÖRFER, Dieter (Hrsg.): Systemtheorie, Systemanalyse und Entwicklungsländerforschung, S. 409-448, Berlin.

EASTON, David 1965: A Systems Analysis of Political Life, New York et al.

GABRIEL, Oscar W. 1975: Einleitung: Strukturprobleme des lokalen Parteiensystems. In: GABRIEL, Oscar W. et al.: Strukturprobleme des lokalen Parteiensystems, S. 1-64, Bonn.

GABRIEL, Oscar W. 1979: Mängelanalyse des politischen Willensbildungsprozesses in der Gemeinde. Ein Beitrag zur "institutionellen Krise" der kommunalen Selbstverwaltung. In: ALBERTIN, Lothar et al.: Politische Beteiligung im repräsentativen System, S. 79-212, Bonn.

GABRIEL, Oscar W. 1984: Kommunalparlament zwischen Parteipolitik und Verwaltung, unveröffentlichtes Manuskript, Mainz.

GABRIEL, Oscar W. 1986: Politische Kultur, Postmaterialismus und Materialismus in der Bundesrepublik Deutschland, Opladen.

Gabriel, Oscar W. (Hrsg.) 1989: Kommunale Demokratie zwischen Politik und Verwaltung, München.

GABRIEL, Oscar W. 1990a: Demokratische Entwicklung und politische Kompetenz. In: Aus Politik und Zeitgeschichte, 40. Jg., Heft B 25, S. 15-26.

GABRIEL, Oscar W. 1990b: Politischer Protest und politische Unterstützung. Entsteht eine neue Subkultur des Protestes in Westeuropa? In: Politische Bildung, 23. Jg., Heft 1, S. 34-52.

GABRIEL, Oscar W. 1991: Das lokale Parteiensystem zwischen Wettbewerbs- und Konsensdemokratie. Eine empirische Analyse am Beispiel von 49 Städten in Rheinland-Pfalz. In: OBERNDÖRFER, Dieter/SCHMITT, Karl (Hrsg.): Parteien und regionale Politische Kultur, S. 371-396, Berlin.

GABRIEL, Oscar W. 1994: Wertewandel, Protestbewegung und Zufriedenheit mit der Kommunalverwaltung in Westeuropa. In: VOIGT, Rüdiger/ GABRIEL, Oscar W. (Hrsg.): Perspektiven der Kommunalwissenschaft, Bochum. 
Gabriel, Oscar W./Haungs, Peter/Zender, Matthias 1984: Opposition in Großstadtparlamenten, Melle.

GRAUHAN, Rolf Richard 1971: Der politische Willensbildungsprozeß in der Großstadt. In: Der Bürger im Staat, 21. Jg., Heft 3, S. 106-111.

GREIFFENHAGEN, Sylvia 1987: Auf den Spuren einer ehemaligen Reichsstadt - Zur Politischen Kultur der Gemeinde Isny im Allgäu. In: BERG-SCHLOSSER, Dirk/SCHISSLER, Jakob (Hrsg.): Politische Kultur in Deutschland. Bilanz und Perspektiven der Forschung. Politische Vierteljahresschrift, Sonderheft 18, S. 267-274, Opladen.

GREIFFENHAGEN, Martin/GREIFFENHAGEN, Sylvia 1981: Ein schwieriges Vaterland. Zur politischen Kultur in Deutschland, Frankfurt am Main.

GREIFFENHAGEN, Martin/GreIFFENHAGEN, Sylvia 1993: Ein schwieriges Vaterland. Zur politischen Kultur im vereinigten Deutschland, München.

GunLICKS, Arthur B. 1986: Local Government in the German Federal System, Durham, Mass.

HAASIS, Hans-Arthur 1979: Entscheidungsstrukturen in der Kommunalpolitik. Eine Bestandsaufnahme der politikwissenschaftlichen Forschung in der Bundesrepublik. In: KÖSER, Helmut (Hrsg.): Der Bürger in der Gemeinde. Kommunalpolitik und politische Bildung, S. 175-199, Bonn.

HÄTTICH, Manfred 1977: Kommunalpolitik - ein politisches Seitengebiet? In: Rausch, Heinz/STAMmEN, Theo (Hrsg.): Aspekte und Probleme der Kommunalpolitik, 3. Aufl., S. 342-354, München.

HALLER, Hans Martin 1979: Die Freien Wähler in der Kommunalpolitik. In: KöSER, Helmut (Hrsg.): Der Bürger in der Gemeinde. Kommunalpolitik und politische Bildung, S. 335-368, Bonn.

HILL, Hermann 1987: Die politisch-demokratische Funktion der kommunalen Selbstverwaltung nach der Reform, Baden-Baden.

HoltmanN, Everhard 1990: Kommunalpolitik im politischen System der Bundesrepublik. In: Aus Politik und Zeitgeschichte, 40. Jg., Heft B 25, S. 3-14.

HoltMANN, Everhard/KILLISCH, Winfried 1989: Gemeindegebietsreform und politische Partizipation. Einstellungen in der fränkischen "Rebellengemeinde" Ermershausen. In: Aus Politik und Zeitgeschichte, 39. Jg., Heft B 30-31, S. 32-39.

INGLEHART, Ronald 1977: The Silent Revolution. Changing Values and Political Styles Among Western Publics, Princeton.

INKELES, Alex 1974: Participant Citizenship in Six Developing Countries. In: BowMAN, Lewis/BoYNTON, Gerald R. (Hrsg.): Political Behavior and Public Opinion. Comparative Analyses, S. 213-247, Englewood Cliffs.

JARREN, Otfried et al. 1989: Lokale Medien und Politische Kultur in Dortmund, Band 10, Begleitforschung des Landes Nordrhein-Westfalen zum Kabelpilotprojekt Dortmund, Düsseldorf.

JAUCH, Dieter 1975: Auswirkungen der Verwaltungsreform in ländlichen Gemeinden, Stuttgart et al. 
KeVENHÖRSTER, Paul (Hrsg.) 1977: Lokale Politik unter exekutiver Führerschaft, Meisenheim am Glan.

KIELMANSEgG, Peter Graf 1989: Auf dem Weg zur demokratischen Reife? Überlegungen zur Entwicklung der politischen Kultur in der Bundesrepublik. In: RUDOLPH, Hermann (Hrsg.): Den Staat denken. Theodor Eschenburg zum Fünfundachtzigsten, S. 199-220, Berlin.

KLAGES, Helmut 1984: Wertorientierungen im Wandel. Rückblick, Gegenwartsanalysen, Prognosen, Frankfurt a.M.New York.

KLAGES, Helmut/HERBERT, Willi 1983: Wertorientierungen und Staatsbezug. Untersuchungen zur politischen Kultur in der Bundesrepublik Deutschland, Frankfurt/New York.

KÖSER, Helmut/CASPERS-MERK, Marion 1989: Einfluß und Steuerungspotential kommunaler Mandatsträger in Baden-Württemberg. In: SCHIMANKE, Dieter (Hrsg.): Stadtdirektor oder Bürgermeister. Beiträge zu einer aktuellen Kontroverse, S. 97-120, Basel et al.

KöSER, Helmut (Hrsg.): Der Bürger in der Gemeinde. Kommunalpolitik und politische Bildung, Bonn.

LeHMan, Edward W. 1971: On the Concept of Political Culture: A Theoretical ReAssessment. In: Social Forces, 50. Jg., Heft 3, S. 361-370.

LEHMBRUCH, Gerhard 1979: Der Januskopf der Ortsparteien. Kommunalpolitik und das lokale Parteiensystem. In: KöSER, Helmut (Hrsg.): Der Bürger in der Gemeinde. Kommunalpolitik und politische Bildung, S. 320-334, Bonn.

LIJPHART, Arend 1980: The Structure of Inference. In: ALMOND, Gabriel A./VERBA, Sidney (Hrsg.): The Civic Culture Revisited. An Analytic Study, S. 37-56, Boston.

MielKE, Gerd/OBERNDÖRFER, Dieter/SCHMITT, Karl (Hrsg.) 1990: Parteien und regionale Politische Kultur, Berlin.

MilbRATH, Lester W./Goel, M. Lal 1977: Political Participation. How and Why Do People Get Involved in Politics? 2. Aufl., Chicago.

NASSMACHER, Hiltrud/NASSMACHER, Karl-Heinz 1979: Kommunalpolitik in der Bundesrepublik. Möglichkeiten und Grenzen, Opladen.

NoHLEN, Dieter 1977: Wahlsysteme und Wahlen in den Gemeinden. In: RAUSCH, Heinz/STAMmEN, Theo (Hrsg.): Aspekte und Probleme der Kommunalpolitik, 3. Aufl., S. 149-186, München.

OEL, Peter 1972: Die Gemeinde im Blickfeld ihrer Bürger. Eine empirisch-soziologische Studie, Stuttgart et al.

PAPPI, Franz-Urban 1970: Wahlverhalten und Politische Kultur. Eine soziologische Analyse der Politischen Kultur in Deutschland unter besonderer Berücksichtigung des Stadt-Land-Unterschiedes, Meisenheim am Glan.

PATRICK, Glenda 1984: Political Culture. In: SARTORI, Giovanni (Hrsg.): Social Science Concepts: A Systematic Analysis, London.

Pross, Helge 1982: Was ist heute deutsch? Wertorientierungen in der Bundesrepublik, Reinbek. 
RIBHEGGE, Wilhelm 1976: Kommunale Selbstverwaltung im Verfassungsstaat. In: FREY, Rainer (Hrsg.): Kommunale Demokratie. Beiträge für die Praxis der kommunalen Selbstverwaltung, S. 28-65, Bonn-Bad Godesberg.

RoHE, Karl 1987: Politische Kultur und der kulturelle Aspekt von politischer Wirklichkeit - Konzeptionelle und typologische Überlegungen zu Gegenstand und Fragestellung politischer Kulturforschung. In: BERG-SCHLOSSER, Dirk/SCHISSLER, Jakob (Hrsg.): Politische Kultur in Deutschland. Bilanz und Perspektiven der Forschung. Politische Vierteljahresschrift, Sonderheft 18, S. 39-48, Opladen.

RosEnBAUM, Walter A. 1975: Political Culture, New York.

REICHEL, Peter 1981: Politische Kultur der Bundesrepublik, Opladen.

RuDZio, Wolfgang 1991: Das politische System der Bundesrepublik Deutschland. Eine Einführung, 3. Aufl., Opladen.

SCHACHT, Konrad 1987: Politische Kultur, sozialer Wandel und Wahlverhalten im Dienstleistungszentrum Frankfurt. In: BERG-SCHLOSSER, Dirk/SCHISSLER, Jakob (Hrsg.): Politische Kultur in Deutschland. Bilanz und Perspektiven der Forschung. Politische Vierteljahresschrift, Sonderheft 18, S. 275-281, Opladen.

SCHÄFER, Peter 1982: Bürgerbefragung Philippsburg 1980. Ein Beitrag zur kommunalen Befragungsforschung, Speyer.

SCHNEIDER, Herbert 1979: Lokalpolitik in einer Landgemeinde. In: KÖSER, Helmut (Hrsg.): Der Bürger in der Gemeinde. Kommunalpolitik und politische Bildung, S. 17-47, Bonn.

SCHUTTEMEYER, Suzanne S. 1986: Bundestag und Bürger im Spiegel der Demoskopie, Opladen.

SIMON, Klaus 1988: Repräsentative Demokratie in großen Städten, Melle.

SONTHEIMER, Kurt 1985: Grundzüge des politischen Systems der Bundesrepublik Deutschland, 10. Aufl., München.

STAMMEN, Theo 1977: Die Erneuerung der kommunalen Selbstverwaltung in Deutschland nach 1945. In: RausCH, Heinz/STAMMEN, Theo (Hrsg.): Aspekte und Probleme der Kommunalpolitik, 3. Aufl., S. 10-32, München.

Verba, Sidney 1978: The Parochial and the Polity. In: PyE, Lucian W./VERBA, Sidney (Hrsg.): The Citizen and Politics. A Comparative Perspective, S. 3-28, Stamford, Conn.

VERBA, Sidney 1980: On Revisiting the Civic Culture. A Personal Postscript. In: ALMOND, Gabriel A./VERBA, Sidney (Hrsg.): The Civic Culture Revisited. An Analytic Study, S. 394-410, Boston.

WAGENER, Frido 1969: Der Neubau der Verwaltung. Gliederung der öffentlichen Aufgaben und ihrer Träger nach Effektivität und Integrationswert, Berlin.

WeHLING, Hans-Georg 1986: Kommunalpolitik in der Bundesrepublik Deutschland, Berlin.

WEHLING, Hans-Georg 1987: Die Bedeutung regionaler politischer Kulturforschung unter besonderer Berücksichtigung Württembergs. In: BERG-SCHLOSSER, Dirk/SCHISSLER, Jakob (Hrsg.): Politische Kultur in Deutschland. Bilanz und Per- 
spektiven der Forschung. Politische Vierteljahresschrift, Sonderheft 18, S. 259-266, Opladen.

WEHLING, Hans-Georg/SIEWERT, H. Jörg 1987: Der Bürgermeister in Baden-Württemberg, 2. Aufl., Stuttgart et al.

ZAHN, Hans-Hermann 1982: Die Einstellungen der Bürger zu ihrer Gemeinde - dargestellt am Beispiel Brackwede-Bielefeld, Baden-Baden.

ZIPFEL, Horst C. 1979: Gemeindeklüngel oder lokale Demokratie? Zur Bestätigung und Widerlegung zweier Klischees. In: KÖSER, Helmut (Hrsg.): Der Bürger in der Gemeinde. Kommunalpolitik und politische Bildung, S. 139-174, Bonn. 